\title{
Differential Patterns of Inputs Create Functional Zones in Central Nucleus of Inferior Colliculus
}

\author{
William C. Loftus, Deborah C. Bishop, and Douglas L. Oliver \\ Department of Neuroscience, University of Connecticut Health Center, Farmington, Connecticut 06030-3401
}

Distinct pathways carry monaural and binaural information from the lower auditory brainstem to the central nucleus of the inferior colliculus (ICC). Previous anatomical and physiological studies suggest that differential ascending inputs to regions of the ICC create functionally distinct zones. Here, we provide direct evidence of this relationship by combining recordings of single unit responses to sound in the ICC with focal, iontophoretic injections of the retrograde tracer Fluoro-Gold at the physiologically characterized sites. Three main patterns of anatomical inputs were observed. One pattern was identified by inputs from the cochlear nucleus and ventral nucleus of the lateral lemniscus in isolation, and these injection sites were correlated with monaural responses. The second pattern had inputs only from the ipsilateral medial and lateral superior olive, and these sites were correlated with interaural time difference (ITD)-sensitive responses to low frequency $(<500 \mathrm{~Hz})$. A third pattern had inputs from a variety of olivary and lemniscal sources, notably the contralateral lateral superior olive and dorsal nucleus of the lateral lemniscus. These were correlated with high-frequency ITD sensitivity to complex acoustic stimuli. These data support the notion of anatomical regions formed by specific patterns of anatomical inputs to the ICC. Such synaptic domains may represent functional zones in ICC.

\section{Introduction}

Auditory processing relies upon brainstem nuclei that process different aspects of sound (Malmierca and Hackett, 2010). The cochlear nucleus $(\mathrm{CN})$, ventral nucleus of the lateral lemniscus (VNLL), and periolivary nuclei code different spectral and temporal aspects of sound but are driven only by sounds in one ear (Malone and Schreiner, 2010; Young, 2010). In contrast, the medial superior olive (MSO), lateral superior olive (LSO), and dorsal nucleus of the lateral lemniscus (DNLL) are "binaural" structures and code interaural time differences (ITDs) or interaural level differences (ILDs) (Yin and Kuwada, 2010).

These monaural and binaural nuclei project to the central nucleus of the inferior colliculus (ICC) with two levels of afferent organization. A primary, "tonotopic" map of best frequency $(\mathrm{BF})$ is created by fibrodendritic laminae (Morest and Oliver, 1984; Oliver and Morest, 1984) and distributed from low frequency dorsolaterally to high frequency ventromedially (Schreiner and Langner, 1997; Malmierca et al., 2005, 2008; Loftus et al., 2008). A secondary "nucleotopic" organization (Brunso-Bechtold et al., 1981) subdivides the ICC into synaptic domains or functional zones that receive inputs from a particular brainstem nucleus or nuclei (Aitkin and Schuck, 1985; Maffi and Aitkin, 1987; Shneiderman and Henkel, 1987; Oliver and Huerta, 1992; Oliver, 2000, 2005; Cant and Benson, 2006).

Received Jan. 19, 2010; revised July 7, 2010; accepted Aug. 4, 2010.

This work was supported by National Institute on Deafness and Other Communications Disorders Grants R01 DC00189 (D.L.0.) and 5 T32 DC00025 (W.C.L.).

Correspondence should be addressed to Douglas L. Oliver, Department of Neuroscience, University of Connecticut Health Center, 263 Farmington Avenue, Farmington, CT 06030-3401. E-mail: doliver@neuron.uchc.edu.

DOI:10.1523/JNEUROSCI.0338-10.2010

Copyright $\odot 2010$ the authors $\quad 0270-6474 / 10 / 3013396-13 \$ 15.00 / 0$
Two pieces of physiological evidence are consistent with the synaptic domain hypothesis. First, the ICC responses often mimic those in subcollicular auditory nuclei (Davis et al., 1999; Ramachandran et al., 1999), suggesting that postsynaptic ICC neurons inherit their properties from ascending inputs. Second, ICC neurons with similar response properties are spatially clustered (Roth et al., 1978; Semple and Aitkin, 1979). Thus, the synaptic domain hypothesis predicts that the segregation of inputs to ICC is an important aspect of the creation of the binaural response properties of ICC neurons. Although the importance of the inputs for neuronal function is assumed, there is seldom a direct test of the hypothesis that a particular pattern of inputs is directly related to a particular response to sound. Our previous reports predict the excitatory inputs from MSO, LSO, and cochlear nucleus will create three separate synaptic domains in ICC with different binaural properties (Oliver et al., 1997; Loftus et al., 2004).

To test this prediction, we combined the evoked responses to sound with retrograde transport and compared the sources of input to the response properties at discrete locations in the ICC. Specifically, we predicted that inputs from MSO would create low-frequency ITD-sensitive ICC neurons, inputs from the cochlear nucleus would produce monaural neurons, and inputs from the contralateral LSO (cLSO) would evoke high-frequency ITD-sensitive and ILD-sensitive neurons. The present results support the first two of these predictions, suggesting a simple relationship between anatomical inputs and monaural or lowfrequency ITD-sensitive ICC sites. However, the third prediction was rejected because the inputs to high-frequency binaural sites received inputs from both MSO and LSO. Nevertheless, the results show a direct correlation between response properties and anatomical inputs. 
These results have been presented in abstract form (Loftus et al., 2006).

\section{Materials and Methods}

Experiments were performed on adult cats (Liberty Laboratories) in accordance with National Institutes of Health guidelines and protocols approved by the Animal Care Committee of the University of Connecticut Health Center (Farmington, CT). The methods used are similar to those published previously (Loftus et al., 2004). Animals were anesthetized with an intramuscular injection of ketamine $(33 \mathrm{mg} / \mathrm{kg})$ and xylazine $(1 \mathrm{mg} / \mathrm{kg})$, intubated, and maintained in an areflexive state with a mixture of isoflurane and medical grade oxygen. They were monitored for reflexive state; breathing rate, heart rate, exhaled $\mathrm{CO}_{2}$, and blood $\mathrm{SpO}_{2}$ were monitored with a SurgiVet capnograph pulse oximeter (V90043, Smiths Medical PM), and body temperature was monitored with a rectal probe coupled to a digital thermometer and maintained at $37^{\circ} \mathrm{C}$ with a water blanket. Lactated Ringer's solution was given intravenously throughout the experiment. The animal was placed in a doublewalled sound attenuation chamber (Industrial Acoustics Company). A craniotomy was performed over the occipital cortex, and a small region of cortex was aspirated to permit visualization of the right inferior colliculus (IC). Glass patch pipettes, pulled to an outer diameter of 3-7 $\mu \mathrm{m}$ and filled with an anatomical tracer dissolved in $0.9 \%$ saline (see below), were used for both recording and injection. The pipette was advanced into the IC, and the neural responses to sound were assessed. Repeated penetrations were made until an appropriate location for the injection was encountered. Following the recording and injections, the muscles and skin were sutured, buprenorphine $(.005 \mathrm{mg} / \mathrm{kg}$, s.c.) was administered for postoperative analgesia, and the animal recovered in an intensive care unit incubator at $37^{\circ} \mathrm{C}$ until fully awake. Recovery from the IC experiments was uneventful, and the animals ate and drank normally a short time after recovering from anesthesia.

Acoustic stimulation, electrophysiological recording, and response analysis. Acoustic stimuli were generated with a TDT System 2 (Tucker Davis Technologies) under the control of a personal computer and were delivered by Beyerdynamic DT-48 earphones via sealed enclosures. Each earphone was coupled to a hollow tube that was held in place by a custom-fitted ear mold (Reprosil, Dentsply International) made for each individual animal. The sound delivery system was calibrated for amplitude and phase between 60 and 40,000 Hz with a half-inch microphone (Brüel and Kjaer) while the ear molds with the embedded sound tubes were inserted in the pinnae. The sound was measured through a narrow probe tube permanently affixed to the interior of the sound delivery tube, and the measured transfer function was corrected for the probe tube characteristics. The amplitude and phase of the acoustic stimulus system were measured as a function of frequency. During sound delivery, we applied an inverse filter (for both amplitude and phase) of the acoustic system to each stimulus. This procedure compensates for the amplitude and phase frequency response of the stimulus system. Our custom software and calibration methods are identical to that used in previous studies (Batra and Fitzpatrick, 2002; Oliver et al., 2003; D’Angelo et al., 2005; Kuwada et al., 2006; Fitzpatrick et al., 2009).

Extracellular recordings of neurons and multiunit clusters were used to localize the ICC and to guide the specific location of the tracer deposit. Search stimuli were broadband noise bursts or pure tones delivered to the contralateral ear or a binaural beat stimulus to both ears (see below). ICC neurons were isolated with a Bak window discriminator (Bak Electronics) and tested for their BF with monaural and binaural pure tones at a level of $60-80 \mathrm{~dB}$ sound pressure level (SPL). The frequency that elicited the greatest response at a given level was deemed the BF. To test ITD sensitivity in neurons with low frequency $(<3 \mathrm{kHz})$ sensitivity, we used a binaural beat stimulus (Kuwada et al., 1979; Batra et al., 1997). This entails a dichotic presentation of two tones with slightly different frequencies (e.g., $1 \mathrm{~Hz}$ ) that produces a cyclic change in the ongoing interaural phase at a rate equal to the difference between the two frequencies. For example, a $1000 \mathrm{~Hz}$ tone to the left ear and a $1001 \mathrm{~Hz}$ tone to the right ear produce a continuously changing interaural phase difference at a rate of $1 \mathrm{~Hz}$ (i.e., the beat frequency). A neuron was identified as ITD sensitive if its response followed the beat frequency. We used a $3 \mathrm{~Hz}$ binaural beat
( $1 \mathrm{~s}$ duration) as a search stimulus and a $1 \mathrm{~Hz}$ binaural beat ( $5 \mathrm{~s}$ duration) as a test stimulus. Some neurons were tested with a $1 \mathrm{~Hz}$ binaural beat stimulus that had identical, $23 \mathrm{~Hz}$ sinusoidal amplitude modulation (SAM) added to the stimulus in both ears (D'Angelo et al., 2003; Sterbing et al., 2003). For neurons with sensitivity to higher frequencies ( $\geq 3 \mathrm{kHz})$, sensitivity to ITD in the envelope of SAM tones was tested with a binaural beat created by slightly disparate modulation frequencies to the two ears (i.e., identical carrier frequency in both ears but the modulation frequencies differed by $1 \mathrm{~Hz}$ ) (Batra et al., 1989, 1993, 1997). ITD sensitivity also was tested by varying the interaural delay of frozen noise or clicks.

ITD sensitivity to binaural beat stimuli was analyzed using methods described previously (Yin and Kuwada, 1983; Kuwada et al., 1989; Fitzpatrick et al., 2000). An individual "delay curve" for each frequency was constructed by plotting the response rate as a function of the ITD. A linear fit of the mean interaural phase of the response as a function of stimulus frequency was used to estimate the neuron's "characteristic delay" (CD) (Rose et al., 1966) based on the slope of the linear fit and "characteristic phase" (CP) based on the $Y$-intercept of the linear fit. The $\mathrm{CP}$ is a measure of whether the delay curves align at the peak (CP near 0 cycles) or trough (CP near 0.5 cycles), and the $\mathrm{CD}$ is an estimate of the difference in conduction times from each ear to the binaural coincidence detector, i.e., the ITD required for coincident arrival of the inputs at each frequency. A single "composite" delay curve for the entire range of tested frequencies was constructed by averaging the individual delay curves.

To test ILD sensitivity, tones with the same frequency were presented to both ears, with the level of the ipsilateral tone varying over successive presentations while the level of the contralateral tone remained constant. In one paradigm, the two tones were presented simultaneously. In another paradigm, a $400 \mathrm{~ms}$ tone was presented to the contralateral ear and a $100 \mathrm{~ms}$ duration tone, delayed by $100 \mathrm{~ms}$, was presented to the ipsilateral ear. With either paradigm, a level-dependent decrease in the firing rate during the ipsilateral stimulus was indicative of ipsilateral ear suppression and ILD sensitivity.

The activity of some neurons was unaffected by any ipsilateral ear input in our battery of test stimuli, and we classified such neurons as "monaural." We cannot exclude the possibility that neurons failing to exhibit binaural sensitivity in the face of our stimulus battery could nevertheless have had latent binaural sensitivity that would have been expressed with a stimulus outside of our tested repertoire (D'Angelo et al., 2003; Sterbing et al., 2003).

Anatomical injections, histology, and analysis of retrograde labeling. Small, focal iontophoretic injections of 3\% Fluoro-Gold (FG) (Fluorochrome) (Schmued and Fallon, 1986; Pieribone and Aston-Jones, 1988) in $0.9 \%$ saline were made at the recording site through the tip of the recording micropipette. In some cases, the FG was mixed with $5 \%$ biotinylated dextran amine (D-1956, Invitrogen) and 5\% fluorescein dextran (D-1820, Invitrogen). Iontophoretic injections were $2 \mu \mathrm{A}$ of $2 \mathrm{~min}$ duration with $7 \mathrm{~s}$ pulses and a $50 \%$ duty cycle ( $7 \mathrm{~s}$ on/7 s off), using a Stoelting precision current source. In initial experiments, we sought to make two injections at sites with similar electrophysiological properties in the same ICC to increase the number of labeled neurons. This strategy proved unnecessary, because we later found that a single injection produced sufficient numbers of retrogradely labeled neurons in the brainstem to yield a clear and unambiguous pattern of labeling. Thus, most of the cases were from a single injection made in the ICC.

Following a 2 week survival period for retrograde transport, animals were deeply anesthetized with ketamine and xylazine and transcardially perfused with $0.12 \mathrm{~m}$ phosphate buffer, $\mathrm{pH} 7.4$, containing $2 \%$ sucrose and $0.05 \%$ lidocaine followed by $4 \%$ formaldehyde in the same buffer. Fixed brains were blocked in the Horsley-Clark plane, and transverse sections through the brainstem were cut at $50 \mu \mathrm{m}$ thickness on a freezing microtome. To view the fluorescent FG-labeled material, one series of sections at $300 \mu \mathrm{m}$ intervals (i.e., every sixth section) were mounted with $\operatorname{DABCO}(1,4$-diazobicyclo-[2.2.2]-octane; Aldrich Chemical) in neutral, buffered glycerine. A second series of sections at $300 \mu \mathrm{m}$ intervals were stained for Nissl substance with cresyl violet and thionin (1:1). A third series of sections at $150 \mu \mathrm{m}$ intervals was used for FG immunohisto- 
Table 1. Percentage of retrogradely labeled neurons from sources in the lower auditory brainstem following injections at physiologically characterized sites in the ICC

\begin{tabular}{|c|c|c|c|c|c|c|c|c|c|c|c|c|c|c|c|c|}
\hline Case & $\mathrm{BF}(\mathrm{kHz})$ & $\begin{array}{l}\text { No. of labeled } \\
\text { neurons }\end{array}$ & $\mathrm{CDCN}$ & CAVCN & CPVCN & $\mathrm{iCN}$ & iVLL & illL & cMSO & iMSO & iLSO & $\mathrm{cLSO}$ & CDLL & iDLL & iP0 & $\mathrm{CPO}$ \\
\hline $144 \square$ & 3 and 6 & 94 & 23 & 24 & 3 & 1 & 37 & 1 & - & - & 1 & - & 7 & 1 & - & - \\
\hline $48 \square$ & 2.7 & 19 & - & 26 & - & - & 74 & - & - & - & - & - & - & - & - & - \\
\hline $36 \square$ & 6.0 & 17 & - & 47 & 12 & - & 24 & - & - & - & - & - & 6 & 12 & - & - \\
\hline 1510 & 0.450 & 122 & - & - & - & - & - & - & - & 98 & 3 & - & - & - & - & - \\
\hline 240 & 0.300 & 61 & - & - & - & - & - & - & - & 93 & 7 & - & - & - & - & - \\
\hline 1760 & 0.350 & 174 & - & - & - & - & - & - & - & 93 & 7 & - & - & - & - & - \\
\hline $34 \triangle$ & 13 & 123 & - & 14 & - & 3 AVCN & 17 & 6 & 2 & 40 & 5 & - & - & - & - & 14 \\
\hline $136 \triangle$ & 15.5 & 500 & - & 14 & - & - & 20 & - & 1 & 10 & 9 & 12 & 1 & 7 & 16 & 11 \\
\hline $39 \triangle$ & 3.7 and 6.5 & 257 & - & - & - & - & 2 & - & - & 37 & 15 & 3 & 17 & 25 & 1 & - \\
\hline $97 \triangle$ & 11 & 273 & - & - & - & $1 \mathrm{DCN}$ & 2 & - & - & 46 & 27 & 21 & - & $<1$ & 3 & 1 \\
\hline $5 \nabla$ & 1.0 & 109 & 1 & - & - & - & 1 & - & 2 & 6 & 6 & 24 & - & - & 59 & 2 \\
\hline
\end{tabular}

Dash (一) indicates no data. Symbols indicate clusters shown in Figure 1.

Table 2. Percentage of retrogradely labeled neurons in periolivary structures

\begin{tabular}{|c|c|c|c|c|c|c|c|c|c|c|c|c|c|}
\hline Case & $\mathrm{BF}(\mathrm{kHz})$ & iVMP0 & cVMPO & iLNTB & cLNTB & iVNTB & cVNTB & CALPO & iDLPO & CDLPO & iDMP0 & cDMP0 & iMNTB \\
\hline $144 \square$ & 3 and 6 & - & - & - & - & - & - & - & - & - & - & - & - \\
\hline $48 \square$ & 2.7 & - & - & - & - & - & - & - & - & - & - & - & - \\
\hline $36 \square$ & 6.0 & - & - & - & - & - & - & - & - & - & - & - & - \\
\hline $151 \bigcirc$ & 0.450 & - & - & - & - & - & - & - & - & - & - & - & - \\
\hline 240 & 0.300 & - & - & - & - & - & - & - & - & - & - & - & - \\
\hline $176 \bigcirc$ & 0.350 & - & - & - & - & - & - & - & - & - & - & - & - \\
\hline $34 \triangle$ & 13 & - & - & - & 11 & - & - & 1 & - & - & - & - & - \\
\hline $136 \triangle$ & 15.5 & 15 & $<1$ & $<1$ & 5 & 1 & $<1$ & - & - & 1 & $<1$ & 3 & $<1$ \\
\hline $39 \triangle$ & 3.7 and 6.5 & - & - & $<1$ & - & - & - & - & $<1$ & - & - & - & - \\
\hline $97 \triangle$ & 11 & 2 & - & - & $<1$ & - & - & 1 & - & - & 1 & - & - \\
\hline $5 \nabla$ & 1.0 & 57 & 2 & - & - & - & - & - & - & - & 2 & - & - \\
\hline
\end{tabular}

Dash (-) indicates no data. Symbols indicate clusters shown in Figure 1.

chemistry. The remaining sections were held in reserve in case additional histology was necessary.

To amplify and preserve the FG signal in a nonfluorescent form, we used immunohistochemical methods. After $20 \mathrm{~min}$ in $0.5 \% \mathrm{H}_{2} \mathrm{O}_{2}$ to remove endogenous peroxidase, sections were rinsed in $0.05 \mathrm{M}$ PBS and exposed to $10 \%$ goat serum with $0.5 \%$ Triton X-100 in PBS for $2 \mathrm{~h}$. Sections were then incubated in anti-FG antibody made in rabbit (Fluorochrome, 1:50,000) in the blocker at $4^{\circ} \mathrm{C}$ overnight. Following PBS rinses, tissue was exposed to a biotinylated anti-rabbit secondary antibody made in goat (Vector Laboratories; $1: 800$ ) in $10 \%$ goat serum blocker for $2 \mathrm{~h}$ at $4^{\circ} \mathrm{C}$. The biotin antibody and the injected biotinylated dextran (if present) were visualized with the ABC-peroxidase method (ABC Elite, Vector Laboratories) performed $2 \mathrm{~h}$ to overnight; and this was followed by preincubation in $0.05 \%$ diaminobenzidine with $0.02 \%$ cobalt chloride and $0.02 \%$ nickel ammonium sulfate $(15 \mathrm{~min})$ and the same solution with $0.005 \% \mathrm{H}_{2} \mathrm{O}_{2}$ (15 min or less). Sections were dried onto subbed slides and coverslipped with Permount (Fisher Scientific).

Neurolucida (Microbrightfield) was used to plot the location of injection sites and retrogradely labeled neuronal somata in complete series of sections with either epifluorescence or light microscopy of the stabilized sections. Nissl-stained sections were used in conjunction with the plotted sections to draw cytoarchitectonic boundaries of IC subdivisions and lower auditory nuclei on both the right and left sides. There were 24 specific structures that are listed in Tables 1 and 2 and comprise the cochlear nuclei, superior olivary nuclei, periolivary nuclei, and nuclei of the lateral lemniscus. The number of labeled neurons in each structure was counted and expressed as a percentage of the total number of labeled neurons in that case. In this way, the anatomical distribution of labeled neurons in a given case was represented by 24 variables, i.e., one variable per structure.

The multivariate anatomical results were analyzed with both cluster analysis and principal components analysis using the MatLab Statistics
Toolbox (Mathworks). The $k$-means algorithm was used to automatically partition the 11 cases into separate clusters on the basis of similarities and differences in the retrograde labeling patterns. The algorithm treats each case as a point in 24-dimensional space and works by minimizing the sum of the squared Euclidean distances from each point to the centroid of its cluster over all clusters. The number of clusters was a user-supplied parameter. We found four clusters to be optimal in terms of providing the best separation among clusters, and the graphs presented are based on this parameter value. A similarity index, a measure of how close a point is to other points in its own cluster relative to points in other clusters, was computed for each point in the partitioned dataset. These measures can range from -1 to +1 and were displayed in a "silhouette plot."

A principal components analysis was done to reduce the high dimensionality of the anatomical data and to visualize the variability in patterns of retrograde labeling. A scree plot, which depicts the fraction of the total variability accounted for by each principal component, indicated that there were no more than three components to be extracted. The anatomical results for all 11 cases were plotted as observations in the lower dimensional space defined by the first three principal components. The score for each principal component is computed by a linear combination of the original 24 anatomical variables, with a different set of coefficients for each principal component. These coefficients (also known as component "loadings") were plotted as vectors with respect to the principal components axes. The scatter plot of observations and the vector plot of coefficients were combined into the same graph, using the "biplot" function of MatLab.

\section{Results}

A total of 13 small focal injections of FG were made in the ICC of 11 animals. The size of the FG deposit was nearly identical across 
all 11 cases, but the different cases exhibited different patterns of retrograde FG labeling. In the dataset as a whole, FG-labeled neuronal somata were found in the cochlear nuclei, the nuclei of the lateral lemniscus, the superior olivary complex, and periolivary nuclei. However, each of the injections produced labeling in only a subset of these structures. Thus, in any given experiment an absence of retrogradely labeled cells in one of these structures is not easily explained by incomplete transport.

A multivariate analysis of the anatomical results suggests there are four patterns of inputs to ICC neurons from the auditory brainstem. The automatic clustering procedure partitioned the 11 cases into four clusters (Fig. 1A) using the 24-dimensional space of anatomical variables (Tables 1 and 2). Table 1 contains the distribution of labeled inputs for all sites in all 11 cases, and Table 2 breaks down the general periolivary categories ( $\mathrm{cPO}$ and $\mathrm{iPO}$ ) into the specific periolivary structures for a total of 24 anatomical measures. There are three clusters of three to four members each plus one "singleton cluster" (Fig. 1A). The first cluster corresponds to sites with the main inputs from contralateral anteroventral cochlear nucleus (cAVCN), dorsal cochlear nucleus (DCN), posteroventral cochlear nucleus, and the ipsilateral VNLL (iVNLL) (Fig. 1A, square; Table 1). The second cluster corresponds to sites whose predominant input is from the MSO, with a slight input from the ipsilateral LSO (iLSO) (Fig. $1 A$, circle; Table 1). The third cluster (Fig. $1 A$, triangle) is a more diffuse cluster, and these four cases have a more varied and complex pattern of inputs than those exhibited by the first two clusters. Finally, the singleton cluster (Fig. $1 A$, inverted triangle) is distinct from the other 10 cases, largely due to its heavy input from the ventromedial periolivary nucleus (VMPO).

The first three principal components collectively account for $92 \%$ of the variability in this anatomical data (Fig. $1 B, C$ ). Individually, the three principal components account for 67.3, 18.6, and $6.1 \%$ of the variability, respectively. The first three cases (Fig. $1 B$, squares labeled 1-3) form a rather tight cluster in the space defined by the first two principal components. This cluster appears to be distinguished mainly by predominant inputs from the cAVCN and iVNLL, judging by its proximity to the coefficient vectors for those two variables. The next three cases (Fig. $1 B$, circles labeled 4-6) form an even tighter cluster on the opposite side of the space and appear to be distinguished by predominant input from MSO. The third cluster is more diffuse and is centered in the principal component space (Fig. $1 B, C$, triangles labeled 7-10), suggesting that this is a more heterogeneous cluster with more complex combinations of inputs. Finally, the singleton cluster (Fig. $1 B, C$, inverted triangle labeled 11) is by itself in the lower part of the space and appears to be distinguished by strong input from the ipsilateral ventromedial periolivary nucleus (iVMPO).

Thus, the anatomical analysis was consistent with the notion of synaptic domains formed by a limited set of inputs to zones in ICC. We next determined the relationship of the response properties at these ICC injection sites to the inputs from the lower auditory brainstem. We predicted that the properties would be related to primary excitatory inputs from cochlear nucleus, MSO, and contralateral LSO.

Sites with convergent input from cochlear nucleus and VNLL The first cluster of cases (Fig. $1 A$, squares) were three experiments $(144,48$, and 36$)$ with injections at the caudal or rostral extremes of the ICC (Fig. 2, squares), and they had similar inputs with FG-labeled neurons found mainly in the contralateral CN and iVNLL (Table 1). At these injections sites, unit activity was
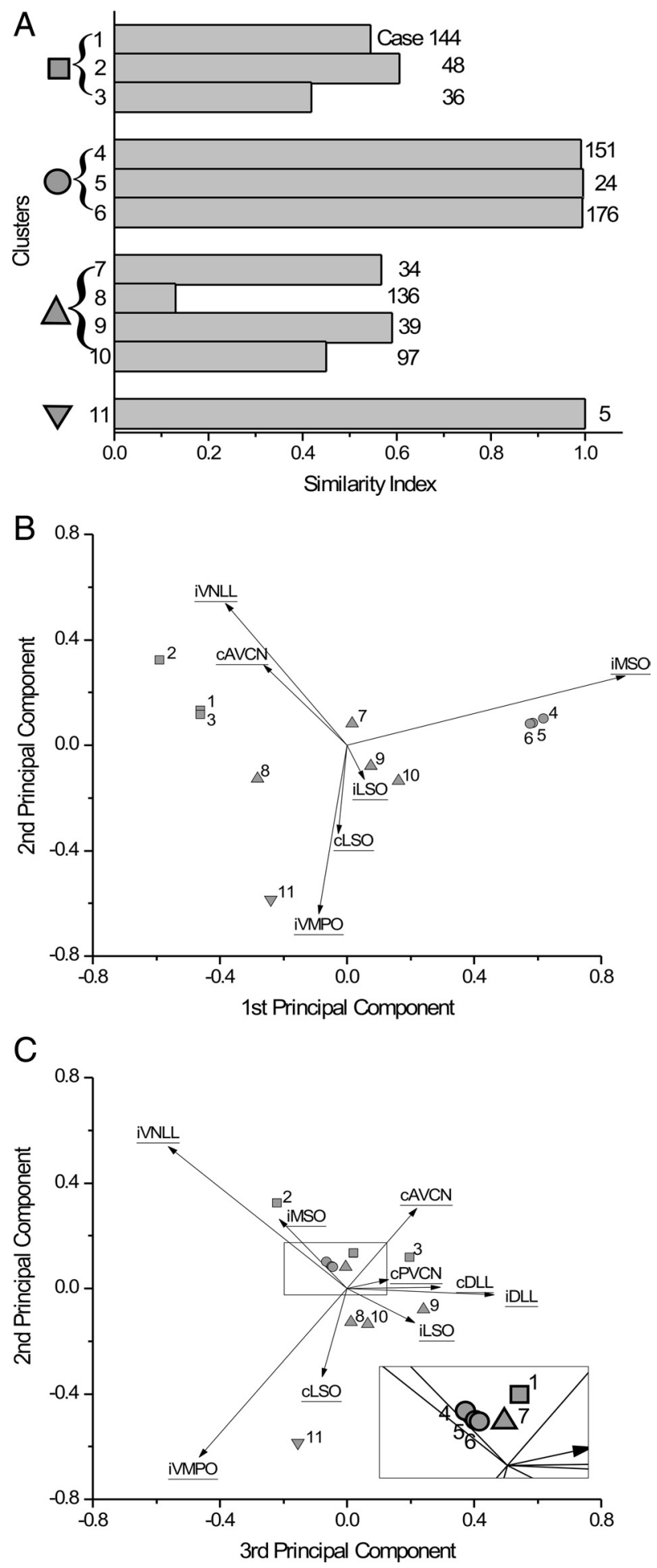

Figure 1. Multivariate analyses of the retrograde labeling data in Tables 1 and 2. A, Silhouette plot of the results of the $k$-means clustering algorithm, which grouped the 11 cases into three clusters plus one singleton cluster. The length of each bar indicates the similarity between that case and the remaining points in the cluster. The case number is indicated at the right of each bar. The symbols and numbers on the left side of the graph correspond to those used in subsequent plots. $\boldsymbol{B}$, Biplot of the scores (symbols) and coefficients (arrows) for the first and second principal components. Each case is indicated by the same symbol/number used in the silhouette plot above. The principal component coefficients/arrows are labeled with the corresponding structure name. Many of the 24 coefficients were too small to be shown on the plot. $\boldsymbol{C}$, Biplot of scores and coefficients for the third and second principal components. Inset shows an enlarged view of data points near the center of the plot. 

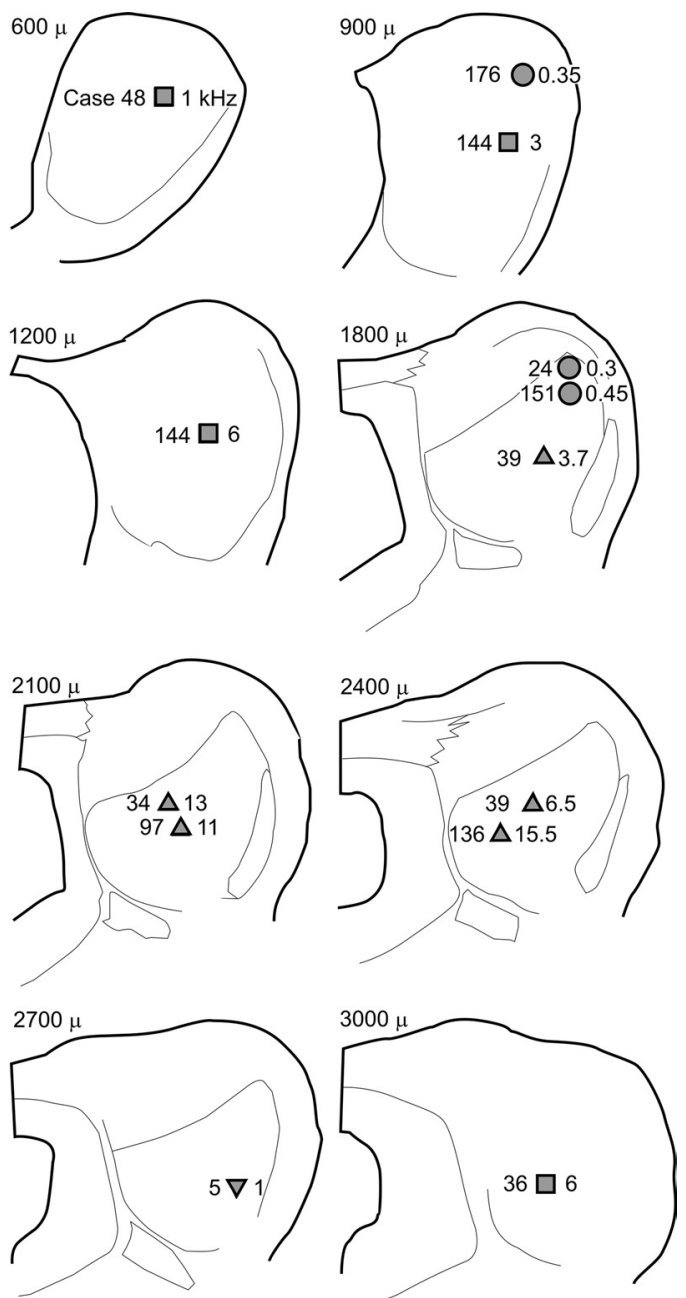

Figure 2.

Figure 2. Composite map of the injection sites for all cases. The locations of the injection site centers of all cases are shown superimposed onto the IC section plots of one case. The symbols correspond to those used in Figure 1. The case number and $\mathrm{BF}$ (in $\mathrm{kHz}$ ) are indicated to the left and right of the symbol, respectively. Sections are arranged from caudal (upper left) to rostral (lower right). Numbers above each section indicate the position (in micrometers) relative to the caudal tip of the IC. Scale bar, $1 \mathrm{~mm}$.

driven only by the contralateral ear and unaffected by simultaneous stimulation to the ipsilateral ear. In these cases, we tested the units extensively with different types of binaural stimuli, including those with amplitude modulations on the envelope or carrier (see Materials and Methods). This usually occupied most of the time the unit was held, and it was not possible to examine responses to all possible monaural stimuli. All three cases had a $\mathrm{BF}$ in the $2.7-6 \mathrm{kHz}$ range. In all three of these monaural sites we verified that there was robust ITD and/or ILD sensitivity elsewhere in the same ICC, far removed from the injected site(s). Thus, the lack of binaural sensitivity reflects a local property of the injected sites. However, the properties and inputs were not identical.

Case 144 had two injection sites with well isolated single units having BFs of $3.5 \mathrm{kHz}$ (Fig. 3A) and $6 \mathrm{kHz}$ (Fig. 3B). Both units failed to show sensitivity to ILD or ITD. Because these units were tuned to high frequencies $(>3 \mathrm{kHz})$, the tests included binaural beats of the modulation frequency of SAM tones. Isointensity frequency sweeps showed that the $6 \mathrm{kHz}$ unit did not broaden its
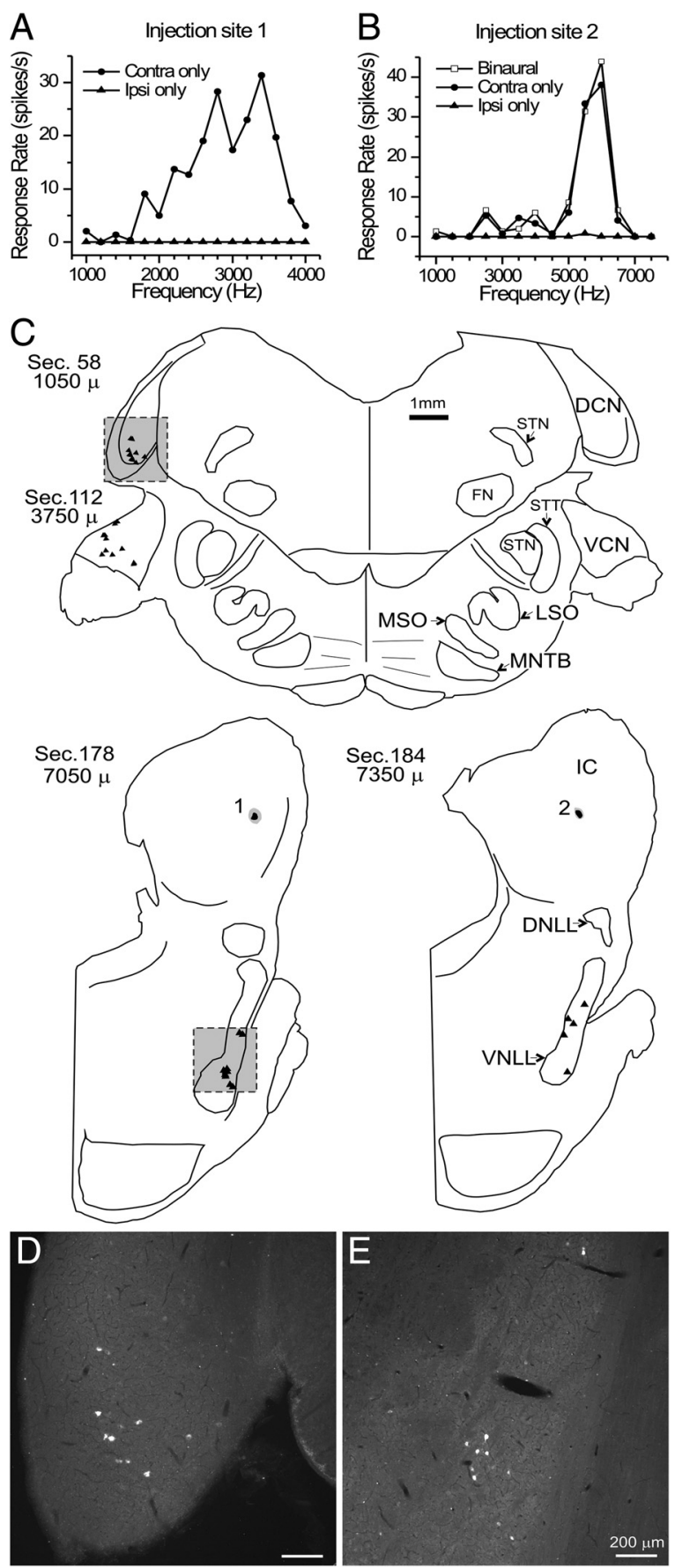

Figure 3. Data for case 144. $A, B$, Frequency tuning for single units recorded at the two different ICC injection sites. Stimuli were presented to the ipsilateral ear only (Ipsi only), the contralateral ear only (Contra only), or diotically, i.e., to both ears (Binaural). Stimulus levels were $60 \mathrm{~dB}$ SPL $(\boldsymbol{A})$ and $65 \mathrm{~dB}(\boldsymbol{B})$. C, Plots of locations of FG-labeled neuronal somata (filled triangles) and IC injection sites in representative sections of the brainstem. Each section is identified by a section number and its position (in micrometers) relative to the caudal margin of the dorsal cochlear nucleus. The right side of the section is ipsilateral to the injected IC. FN, Facial nucleus; STN, spinal trigeminal nucleus; STT, spinal trigeminal tract. See Results for abbreviations of the auditory structures. $\boldsymbol{D}, \boldsymbol{E}$, Photomicrographs (epifluorescence) of the boxed areas in Cshowing retrogradely labeled neuronal somata in the $\mathrm{CN}(\boldsymbol{D})$ and the VNLL $(\boldsymbol{E})$.

tuning with intensity increases. The $Q_{10}$ of 4 , the $Q_{40}$ of 2 , and the $\mathrm{BF}$ above $3 \mathrm{kHz}$ suggest that the unit is an I-type neuron (Ramachandran et al., 1999). The $3.5 \mathrm{kHz}$ unit had an onset response, a $Q_{10}$ of 1.75 , and a $Q_{40}$ of 1.16 , but it is not clear whether this is a V-type or I-type since the BF and $\mathrm{Q}$ values 


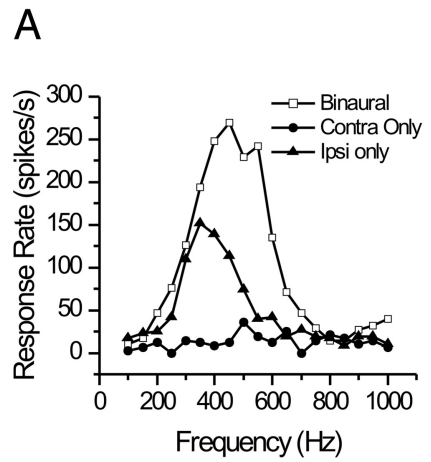

B
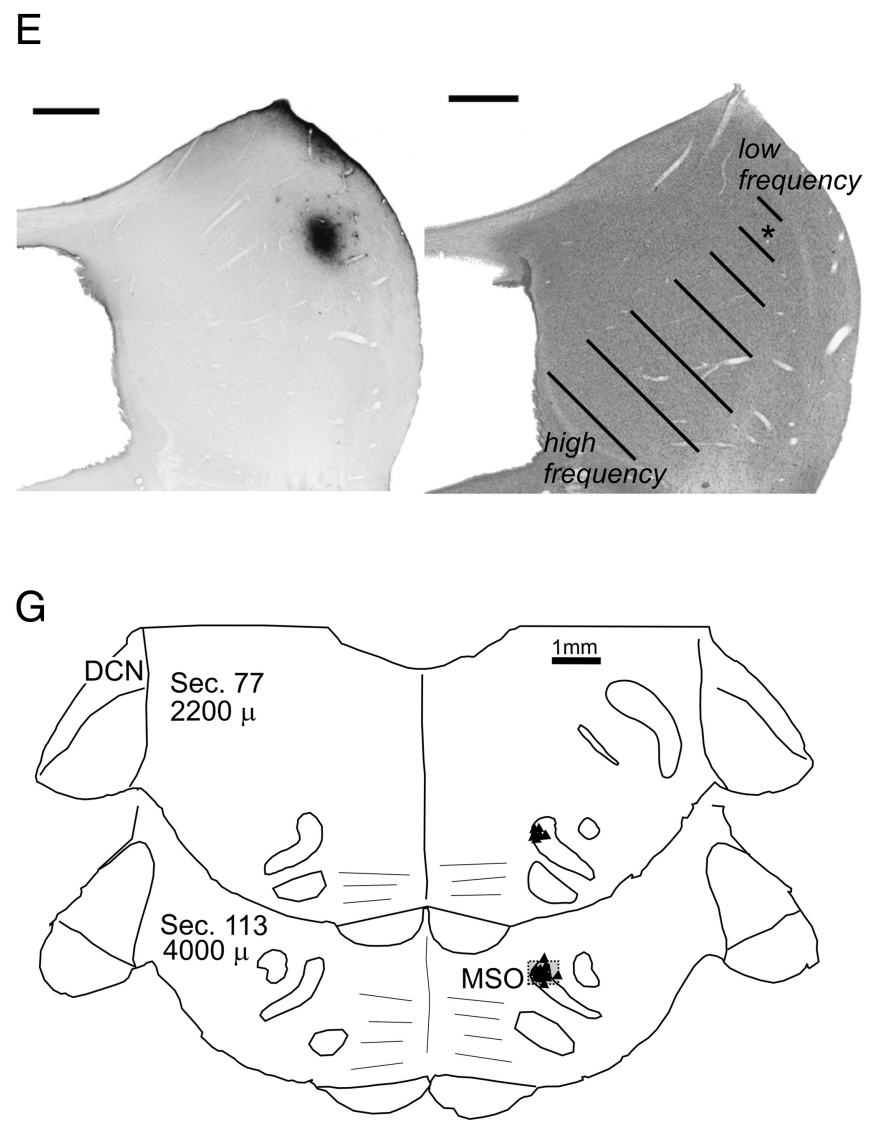
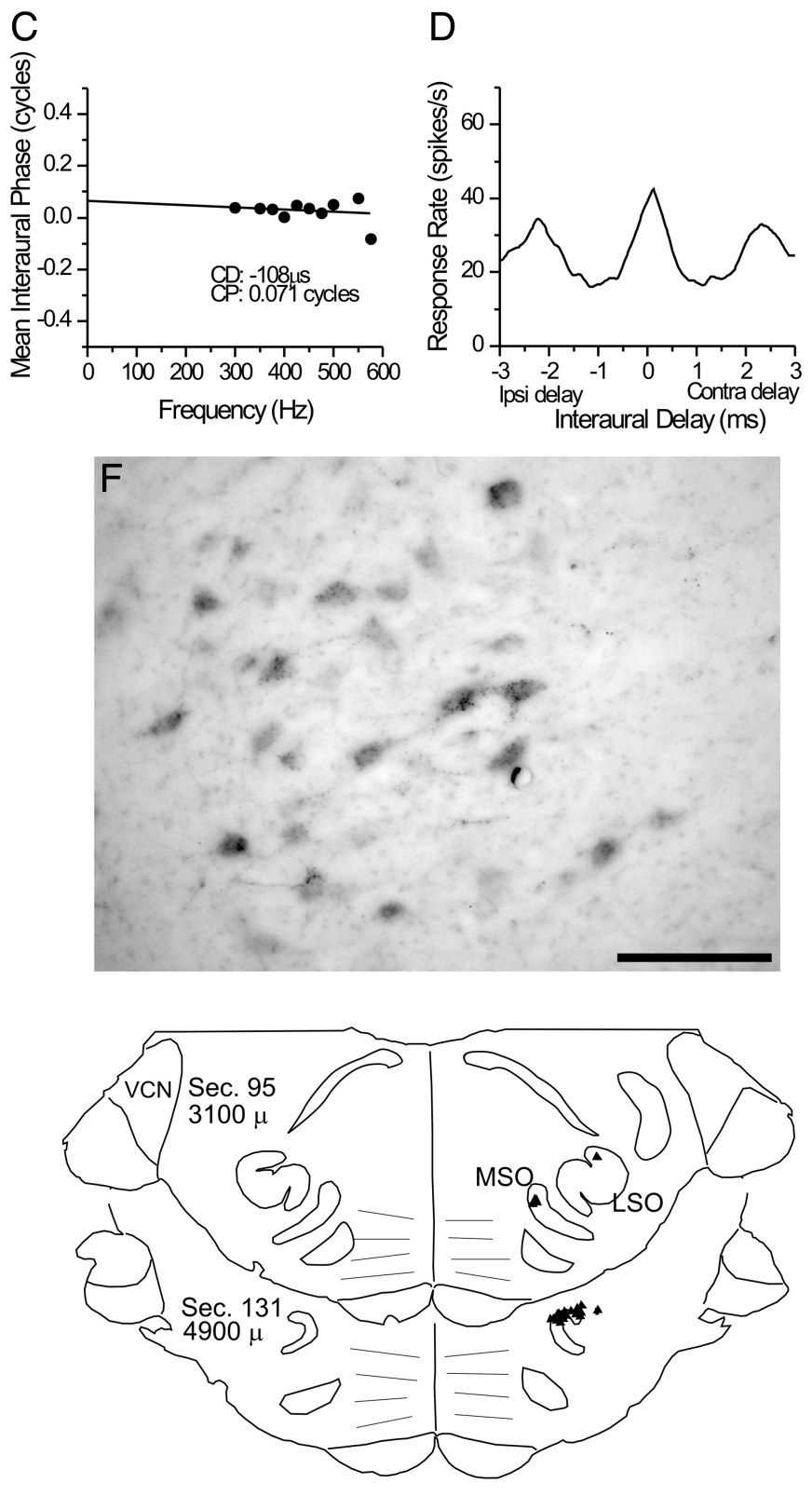

Figure 4. Data for case 151. A-D. Multiunit responses recorded at the injection site. $\boldsymbol{A}$, Frequency tuning for stimuli at $80 \mathrm{~dB}$. $\boldsymbol{B}$, ITD tuning curves obtained with a $1 \mathrm{~Hz}$ binaural beat stimulus with the "beat" on the carrier frequency. Tested frequencies were between 300 and $550 \mathrm{~Hz}$, in $50 \mathrm{~Hz}$ steps. $\boldsymbol{C}$, Phase versus frequency plot. $\boldsymbol{D}$, Composite ITD tuning curve. $\boldsymbol{E}$, Injection at the ICC recording site in and nearby Nissl-stained section (right). The asterisk indicates the position of the injection site center; black lines indicate the orientation and progression of frequency band lamina in the ICC. $\boldsymbol{F}$, Photomicrograph (immunohistochemical staining) of retrogradely labeled neuronal somata in the dorsal MSO. $\boldsymbol{G}$, Plots of retrogradely labeled neurons in representative sections. The box in section (Sec.) 113 corresponds to the area shown in $\boldsymbol{F}$. Scale bars: $\boldsymbol{E}, \mathbf{G}, 1 \mathrm{~mm} ; \boldsymbol{F}, 20 \mu \mathrm{m}$.

overlap those seen for both categories (Ramachandran et al., 1999). The FG-labeled neurons were found in all three divisions of the cochlear nucleus in isolated, low-density patches (Fig. 3C$E)$. However, we did not see two separated bands of retrogradely labeled cells in the cochlear nucleus even though the injection sites were separated by one octave and were not in the same ICC section (Fig. 3C). Most cells were in the anteroventral cochlear nucleus (AVCN) and the DCN. Patches of labeled cells were also seen in VNLL, where tonotopic organization is more difficult to visualize (Merchan and Berbel, 1996).

Injected sites in the other two cases also failed to respond to binaural stimuli. Case 48 had a monotonic rate level function up to $80 \mathrm{~dB}$ and a $Q_{20}$ of 1.4 , consistent with either an I-type or a $\mathrm{V}$-type response. Case 36 had a saturating rate level function, responding up to $80 \mathrm{~dB}$, and $Q_{20}$ of 2.4. These data and the $B F$ of case 36 suggest that the unit was an I-type. Both cases showed retrograde labeling primarily limited to the AVCN and VNLL.

\section{Sites dominated by MSO inputs}

The second cluster of cases (Fig. $1 A$, circles, 151, 24, and 176) had injections at ITD-sensitive sites in the low-frequency, dorsolateral ICC (Fig. 2, circles). All three cases exhibited the same overall retrograde pattern, with the vast majority of FG-labeled neurons in the MSO and the remaining small proportion of labeled neurons in the ipsilateral LSO (Table 1).

The multiunit response at the injection site in case 151 was a peak-type ITD response [Kuwada et al. (1989), their Fig. 5]. The binaural BF was $450 \mathrm{~Hz}$ at $80 \mathrm{~dB}$ (Fig. 4A). The BF for the ipsilateral ear was somewhat lower in frequency, and the response to 
the contralateral ear was too weak to determine a BF. The response to the binaural beat stimulus was strongly modulated by ITD as shown in the delay curves taken at a different frequency (Fig. $4 B$ ). The peaks of the delay curves clustered near zero, and the interaural phase versus frequency plot intersected the $y$-axis near 0 cycles (Fig. 4C). The peak of the response in the composite delay curve (Fig. 4D) indicates a best delay of $120 \mu$ s. Consistent with the $\mathrm{BF}$, the injection site indicated that this recording was in the dorsolateral ICC (Fig. 4E). Almost all of the retrogradely FG-labeled neurons in the brainstem of this case were confined to the ipsilateral MSO (Fig. 4F, G; Table 1) with the few remaining neurons in the low-frequency, dorsolateral limb of the ipsilateral LSO. In transverse sections, the neurons were clustered in the dorsal third of the MSO. This pattern is consistent with the tonotopic gradient of the MSO (low to high frequencies in a dorsoventral direction) and the low-frequency BF of the ICC injection site. The distribution of neurons along the rostral-caudal axis of the MSO was more or less uniform, and neurons were found in all sections throughout the MSO, consistent with multiple MSO inputs converging onto a single ICC site as opposed to a simple point-to-point pattern (Oliver et al., 2003).

A somewhat more complex response was seen at the injection site of case 24, where a multiunit recording showed no response to a pure tone binaural beat (Fig. $5 A$, open squares). However, a response was evoked by monaural SAM tones in either ear (Fig. $5 A$, filled circles and triangles), and an unambiguous response to low-frequency ITDs was evoked by a $1 \mathrm{~Hz}$ binaural beat with a fixed modulation frequency of $23 \mathrm{~Hz}$ on the carrier frequencies between 100 and $500 \mathrm{~Hz}$ (Fig. 5A, filled squares). ITD sensitivity to the carrier frequency of SAM tones was reported previously in the ICC of the rabbit (D'Angelo et al., 2003; Sterbing et al., 2003). Under these conditions, the response to contralateral stimulation was stronger than that to ipsilateral stimulation, and both showed BFs near $200 \mathrm{~Hz}$ (Fig. 5A). A single unit was also well isolated at this same site (Fig. $5 B-D$ ). The interaural phase versus frequency plot for this unit was nonlinear. Most of the higher frequencies had a characteristic phase near 0 cycles (Fig. $5 B$, circles, solid line), and the composite ITD curve for the same frequencies indicated a peak-type response (Fig. $5 C$ ). However, at frequencies below $350 \mathrm{~Hz}$ the phase shifted away from zero (Fig. $5 \mathrm{~B}$, triangles, dotted line), and there was a decrease and shift in the response near 0 ITD (Fig. 5D). What may be more important than the unusually large characteristic delay is that within the physiological range of ITDs, the activity shifts from one side of 0 ITD to the other as the stimulus frequency drops below $350 \mathrm{~Hz}$ (Fig. 5B-D). This nonlinear response is consistent with a convergence of two excitatory inputs with different ITD sensitivity on the same ICC neuron. As in the case above, all of the FG-labeled neurons were in the ipsilateral superior olivary complex, with most (93\%) in the dorsal MSO (Fig. 5Eb) and the remainder (7\%) in the dorsolateral tip of the LSO (Fig. $5 E a$ ). Thus, these anatomical data suggest that for this unit the ipsilateral MSO was one source of excitatory ITD-sensitive input, and the LSO was a second source of excitatory input. Because LSO neurons often fire at interaural delays near $\pm 2 \mathrm{~ms}$ (Joris and Yin, 1995), an excitatory input from LSO may explain the large nonzero response at lower frequency.

Like the previous case, case 176 also shows evidence of convergence from MSO and LSO onto a site with a low BF (Fig. 6A). This unit also had unambiguous ITD sensitivity to low frequencies and a nonlinear interaural phase versus frequency plot (Fig. $6 B$ ). From 450 to $700 \mathrm{~Hz}$ the CPs indicate a common phase delay around 0.5 cycles, typical of a trough-type response. This is consistent with the composite curve that had the predicted trough-
A
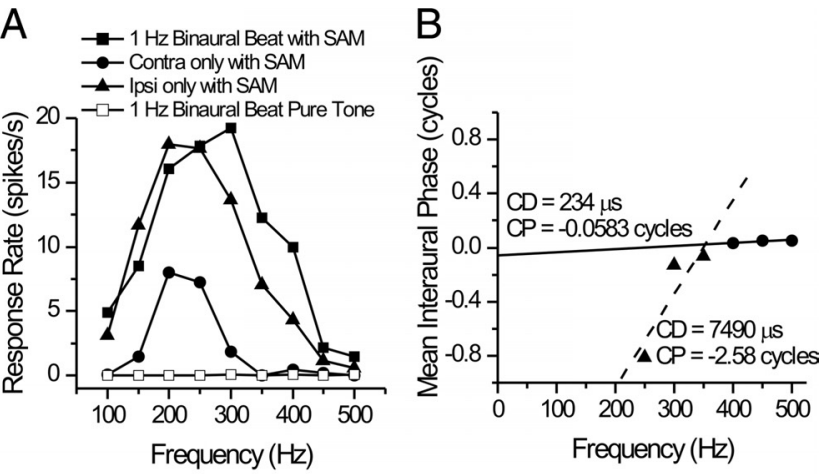

C

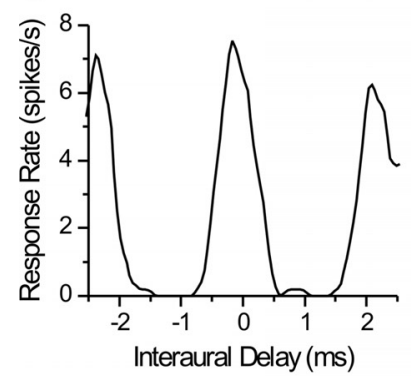

D

\section{E}

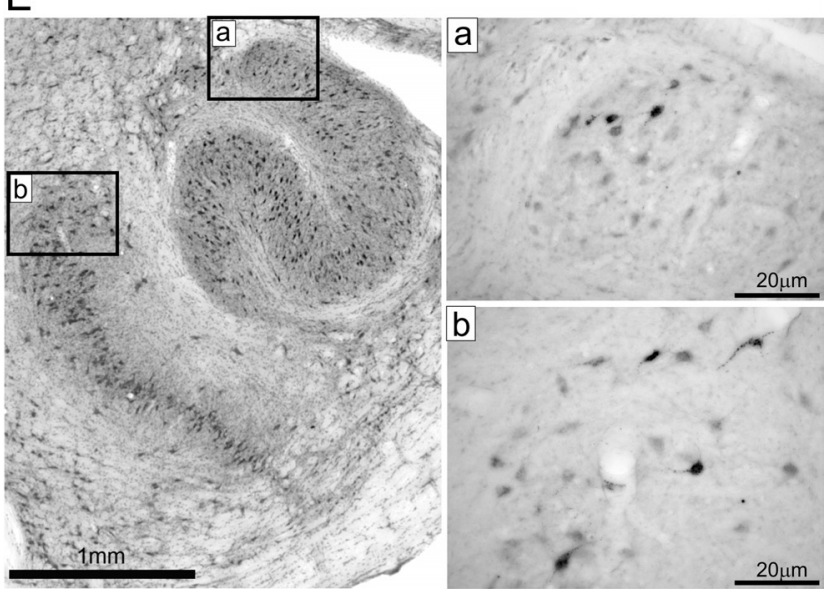

Figure 5. Data for case 24. $\boldsymbol{A}$, Frequency tuning of a multiunit recording at the ICC injection site. $\boldsymbol{B}-\boldsymbol{D}$, Response data of a well isolated single unit at the same site. Data were collected with a $1 \mathrm{~Hz}$ binaural beat with an identical $23 \mathrm{~Hz}$ SAM added to the stimulus in both ears. $\boldsymbol{B}$, Phase versus frequency plot. Delay tuning was heterogeneous across frequency. The unit had a definite peak-type response for frequencies between 400 and $500 \mathrm{~Hz}$ (circles, solid line). Delay tuning deviated from this dramatically at lower frequencies ( $\leq 350 \mathrm{~Hz}$, triangles, dashed line) with trough-like characteristics $C, D$, Composite delay curves, one for each frequency range, are shown in $\boldsymbol{C}(\geq 400 \mathrm{~Hz}$ ) and $\boldsymbol{D}(\leq 350 \mathrm{~Hz})$. $\boldsymbol{E}$, Nissl-stained section (left) and immunohistochemical photomicrographs (right) through the superior olivary complex ipsilateral to the injected ICC. Boxes ( $\boldsymbol{a}$ and $\boldsymbol{b}$ ) on the Nissl section indicate the cytoarchitectonic location of FG-labeled neuronal somata in the dorsolateral LSO $(\boldsymbol{a})$ and the dorsal MSO $(\boldsymbol{b})$ from adjacent immunoreacted sections.

type shape (Fig. 6D). However, the phase-versus-frequency plot of the lower frequency range $(\leq 400 \mathrm{~Hz})$ indicates a phase delay of 0.7 cycles, which is in between the 0 cycles of a "typical" peak response and the 0.5 cycles of a "typical" trough response (Fig. $6 B)$. The composite curve in the lower frequency range showed both a prominent peak slightly below $0 \mathrm{~ms}$ and a trough above 1 ms delay (Fig. 6C), indicating once more that there was a shift in the response within the physiological range of ITDs at higher versus lower frequencies. The proportions of labeled inputs in the 
A
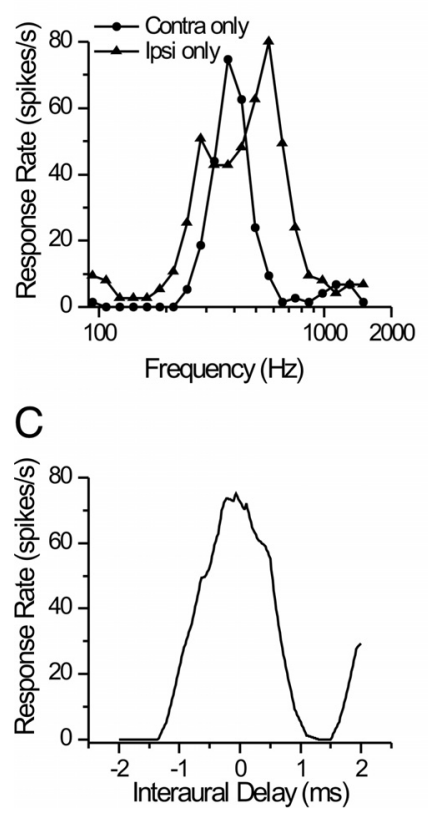

B

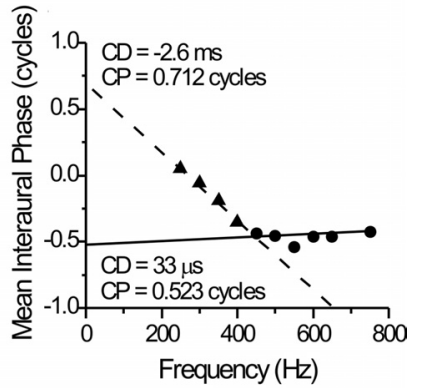

D

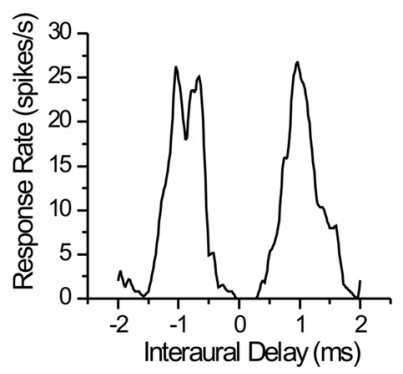

Figure 6. Data for case 176. $\boldsymbol{A}$, Frequency tuning of a single unit at the ICC injection site. $\boldsymbol{B}$, Phase versus frequency plot. The unit had peak-type response for frequencies $\leq 400 \mathrm{~Hz}$ (triangles, dashed line) and a trough-type response for frequencies between 450 and $750 \mathrm{~Hz}$ (circles, solid line). C, Composite delay curve for frequencies $\leq 400 \mathrm{~Hz}$. D, Composite delay curve for frequencies $\geq 450 \mathrm{~Hz}$.

MSO and ipsilateral LSO were identical to the previous case (Table 1), and they also support the convergence of excitatory inputs.

\section{Sites with multiple olivary inputs}

The third cluster of four cases (Fig. 1 A, upright triangles, 34, 136, 39 , and 97) had injection sites distributed around the central area of the ICC, both in terms of location along the rostral-caudal axis and the dorsolateral-ventromedial frequency axis (Fig. 2, upright triangles). This cluster was less consistent in the pattern of inputs (Table 1). All had prominent inputs from the ipsilateral MSO and additional inputs including, but not limited to, the contralateral MSO, contralateral LSO, or contralateral cochlear nucleus. These injected neurons exhibited complex forms of ITD sensitivity. In general, they responded to ITDs in envelopes, clicks, noise, or amplitude modulated stimuli, but not to ITDs in pure tones. Somewhat surprisingly, most did not respond to ILDs, even if they had significant LSO input.

Two cases (34 and 136) had significant input from both superior olive and AVCN (Table 1). Consistent with a BF between 13 and $16 \mathrm{kHz}$, the injection sites were at ICC sites located in the ventromedial part of the ICC (Fig. 2, triangles). Interestingly, they both shared sensitivity to ITDs in the envelopes of SAM tones.

The neuron in case 34 showed both ITD and ILD sensitivity. The ILD sensitivity is illustrated by the inhibition of the response by sound in the ipsilateral ear when it was inserted $100 \mathrm{~ms}$ after the start of the contralateral tone (Fig. 7A). At $80 \mathrm{~dB}$ SPL, the ipsilateral stimulus completely suppresses the response to the contralateral stimulus, and at $60 \mathrm{~dB}$ SPL the suppressive effects of the ipsilateral tone can no longer be detected. Both pure tones in Figure $7 A$ were at the $\mathrm{BF}$ of $13 \mathrm{kHz}$ (Fig. $7 B$ ). ITD sensitivity for this neuron to the envelope of a SAM tone is shown by the trough-type composite delay curve with firing minima at an ITD of $2 \mathrm{~ms}$ (Fig. 7C). The phase versus frequency plot is consistent

A

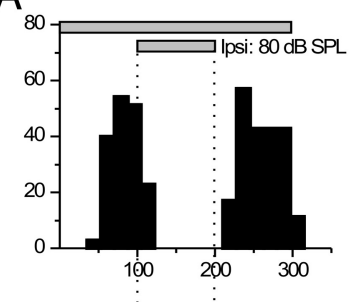

B
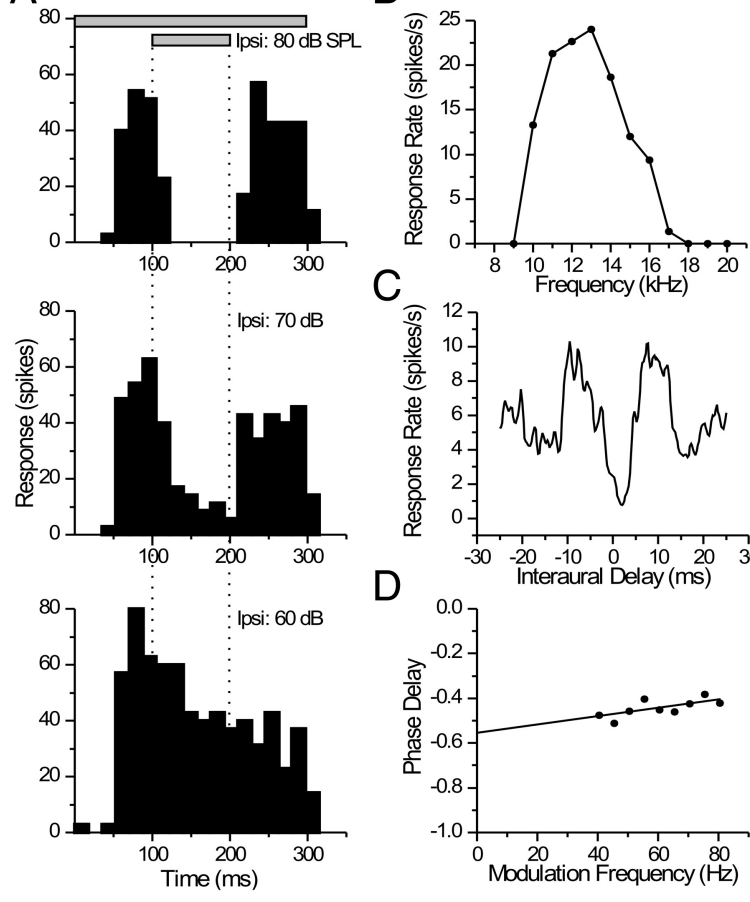

C
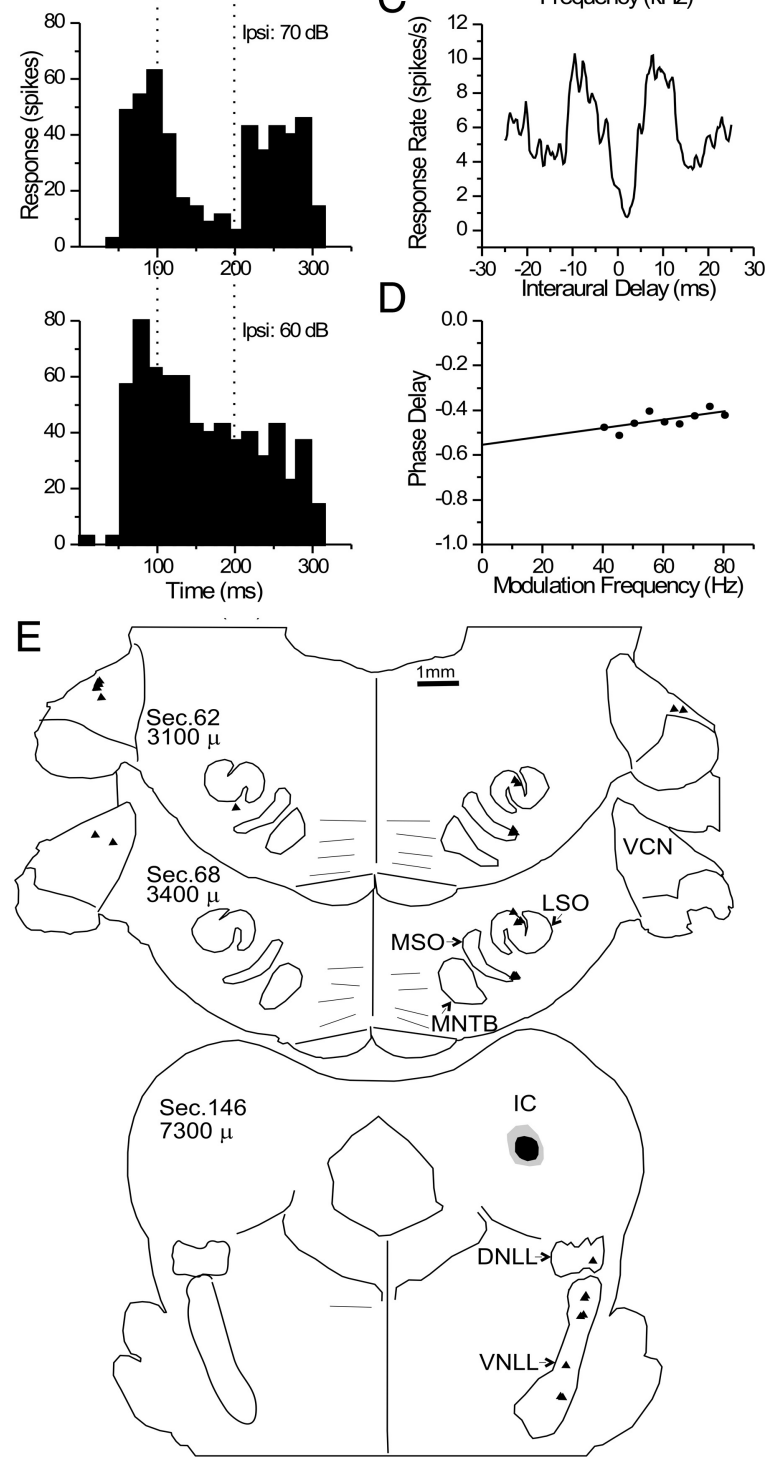

Figure 7. Data for case 34. A, Poststimulus time histograms (PSTNs) for a single unit at the injection site, indicating suppression by the ipsilateral ear. The contralateral ear stimulus was $300 \mathrm{~ms}$ in duration and was initiated at time 0 on the PSTHs. The ipsilateral stimulus was $100 \mathrm{~ms}$ in duration and was delayed $100 \mathrm{~ms}$ relative to the onset of the contralateral ear stimulus. The level of the ipsilateral stimulus is indicated in each histogram (the contralateral stimulus was constant at $60 \mathrm{~dB} \mathrm{SPL}$ ). The dashed lines indicate the onset and offset of the ipsilateral ear stimulus. $B$, Frequency tuning for SAM stimuli (23 Hz modulation) delivered to the contralateral ear at $70 \mathrm{dBSPL}$. C, Composite delay tuning curve obtained with $1 \mathrm{~Hz}$ binaural beat stimuli, with the beat on the modulation frequency. The carrier frequency was $14 \mathrm{kHz}$. The composite combines delay curves obtained with modulation rates from $40 / 41 \mathrm{~Hz}$ to $80 / 81 \mathrm{~Hz}$, in $5 \mathrm{~Hz}$ steps. D. Phase versus frequency plot for the nine tested modulation frequencies. $\boldsymbol{E}$, Plots of retrogradely labeled neurons in representative sections (Sec.) and the ICC injection site.

with this trough shape and intersects the $y$-axis at a CP near 0.5 cycles (Fig. 7D) [Batra et al. (1993), their Fig. 2)]. Both ILD sensitivity and ITD sensitivity could be related to inhibition produced by glycinergic inputs from the ipsilateral LSO in the ICC 
A

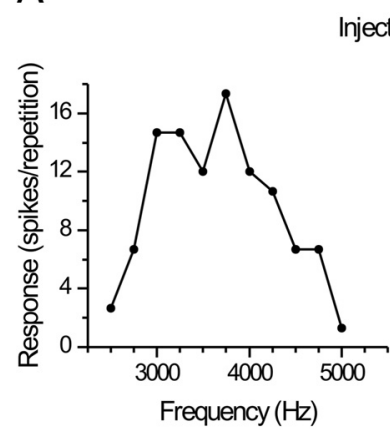

B

Injection site 1

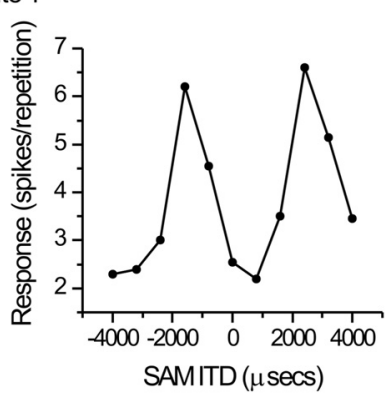

C

$\mathrm{D}$
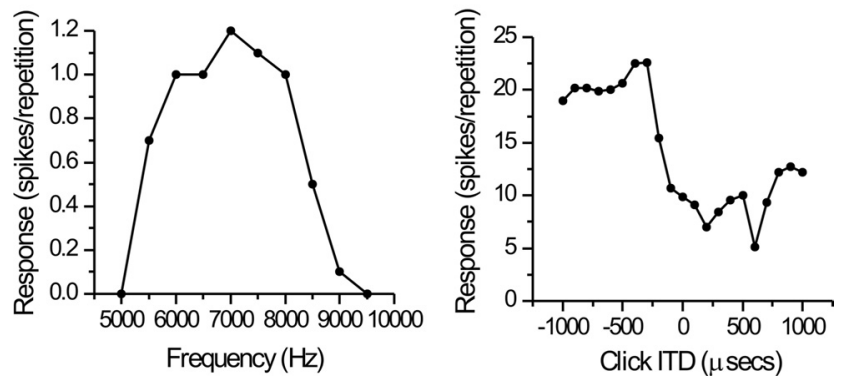

Figure 8. Data for case 39. $\boldsymbol{A}$, Frequency tuning of a single unit at injection site 1. Stimuli were diotic tones delivered at $60 \mathrm{dBSPL}$. $\boldsymbol{B}$, Interaural delay tuning of the same unit, tested with an amplitude modulated tone $(250 \mathrm{~Hz})$ and a $3750 \mathrm{~Hz}$ carrier. C, Frequency tuning of a multi unit at injection site 2. Stimuli were tones delivered at $60 \mathrm{~dB}$ SPL to the contralateral ear. $\boldsymbol{D}$, Interaural delay tuning at injection site 2 , with click stimuli.

(Fig. 7E; Table 1). However, the largest input to this site was from the ventral, high-frequency part of the MSO (43\%) (Fig. 7E). Little is known about the response of higher frequency MSO neurons. Whether high-frequency MSO is ITD sensitive and whether it receives inhibitory inputs driven by the ipsilateral ear are both important unresolved factors in this case. In any event, the data suggest that the response reflects a convergence of ITDsensitive and monaural (AVCN, VNLL) inputs.

A similar situation is seen in case 136 (Fig. 2, triangle) where a neuron with a $\mathrm{BF}$ of $15 \mathrm{kHz}$ in ICC was ITD sensitive to the envelope of a SAM tone and had a peak-type delay curve with a best ITD at $125 \mu$ s (data not shown). Here, the binaural inputs arose from the MSO and bilaterally from LSO and DNLL. As before, these binaural inputs converged with monaural inputs from the AVCN, VNLL, and two periolivary nuclei: the ventromedial periolivary nucleus on the ipsilateral side, iVMPO, and the lateral nucleus of the trapezoid body on the contralateral side, cLNTB (Tables 1, 2).

Two ITD-sensitive sites were injected in case 39 (Fig. 8). These were lower in $\mathrm{BF}$ and near the center of the ICC (Fig. 2, triangles). At the $3.75 \mathrm{kHz}$ site (Fig. $8 \mathrm{~A}$ ), neurons also failed to show ILD sensitivity and exhibited no response to pure tones in the ipsilateral ear. However, once again, they were sensitive to ITDs in the envelopes of SAM tones (carrier, $3750 \mathrm{~Hz}$; modulation frequency, $250 \mathrm{~Hz}$ ) (Fig. 8 B). The second site had neurons with BFs near $7 \mathrm{kHz}$ (Fig. 8C) and had an onset-only response to all tested stimuli. Because it did not have a sustained response to binaural beat stimuli, we could not elicit envelope-ITD sensitivity in this manner. However, neurons at this site were sensitive to ITDs in clicks (Fig. 8D). The inputs to these sites were similar to those above, with an array of MSO and LSO inputs. However, the DNLL inputs were more prominent, and the AVCN inputs were lacking.
A
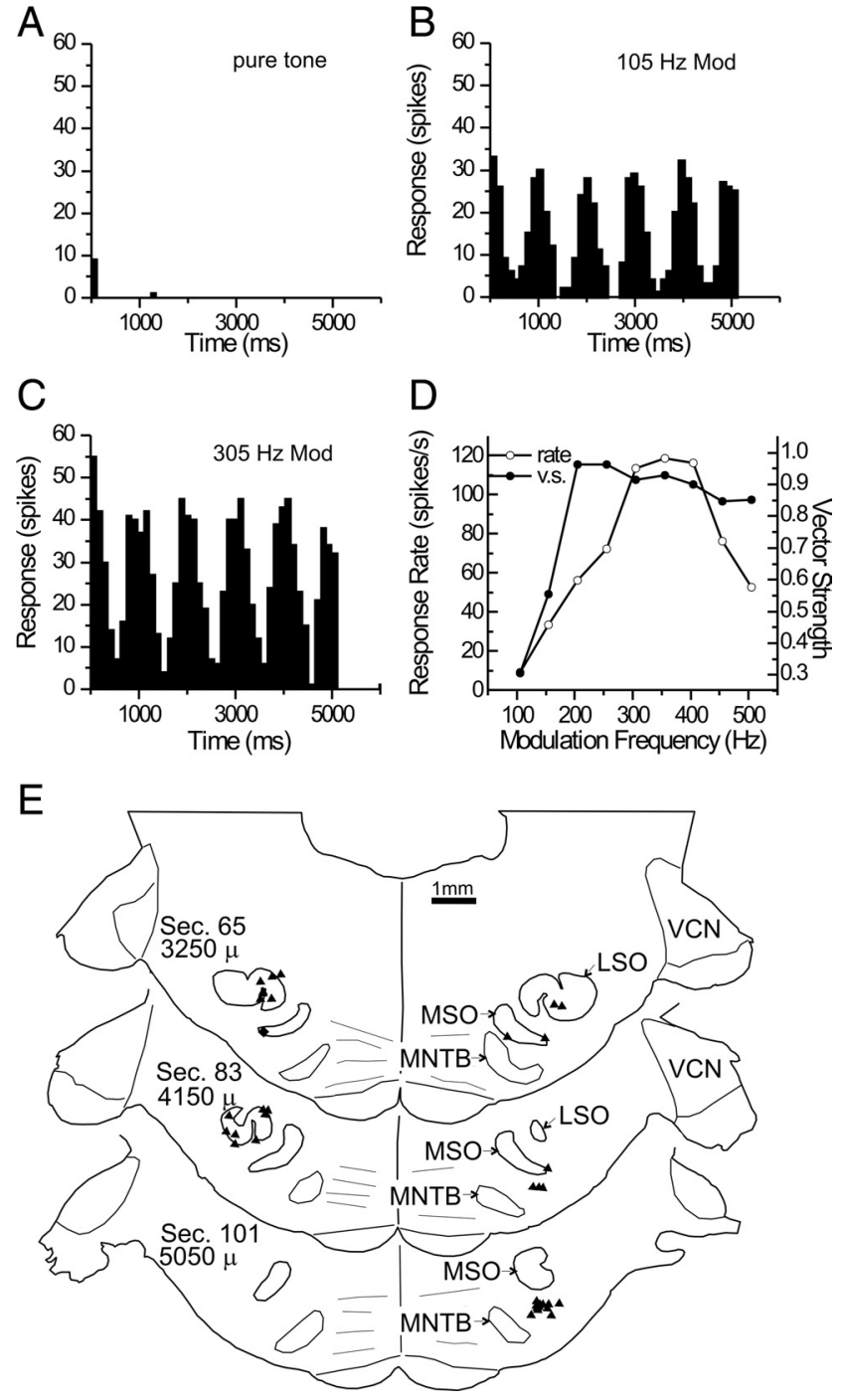

Figure 9. Data for case 5. $\boldsymbol{A}-\boldsymbol{C}$, PSTHs for a $1 \mathrm{~Hz}$ binaural beat on a $1000 \mathrm{~Hz}$ carrier. The modulation rate is indicated in each histogram. $\boldsymbol{D}$, Modulation transfer functions in terms of response rate and synchrony/vector strength. $\boldsymbol{E}$, Plots of retrogradely labeled neurons in representative sections.

A final case in this group, case 97 (Fig. 2, triangle), had an 11 $\mathrm{kHz}$ BF site in ICC that was excited by frozen noise in either ear at higher intensity levels (data not shown). Below $80 \mathrm{~dB}$, the unit was not modulated by tonal or noise stimuli in the ipsilateral ear. Consequently, the ITD sensitivity was not tested. As above, most inputs were from the superior olivary complex, with minimal input from the cochlear nucleus and VNLL (Table 1).

\section{A final complex olivary case}

The final cluster is represented by a single case, case 5 (Fig. 1, inverted triangle). At this site in rostrolateral ICC (Fig. 2, inverted triangle), a single unit with a $\mathrm{BF}$ of $1 \mathrm{kHz}$ responded to tones only with an onset response (Fig. 9A). However, the unit exhibited ITD sensitivity in response to a $1 \mathrm{~Hz}$ binaural beat on the carrier of a SAM tone (Fig. 9B, C) (both ears received the same modulation frequency). This unit was also selective for modulation rate, as shown by the modulation transfer functions (Fig. 9D). The rate modulation transfer function was bandpass with a peak at 355 Hz. In contrast, the synchrony modulation transfer function was high pass and had reached maximum near $200 \mathrm{~Hz}$. This site was 
distinguished in the cluster analysis (Fig. 1), because the largest single input was from ipsilateral VMPO (Fig. 9E). As in the previous group, the other inputs were from the superior olivary complex, but the predominant input was from the contralateral LSO rather than the input from the MSO and ipsilateral LSO.

\section{Discussion}

The present results show that small, localized regions to the ICC differ in their inputs from the lower auditory brainstem. These regions contained neurons with different types of ITD sensitivity. However, we did not find the predicted, relatively simple pattern of synaptic domains defined by separate excitatory inputs from contralateral cochlear nucleus, LSO, and ipsilateral MSO. Instead, we found at least three patterns of anatomical inputs that appear to be correlated to different types of binaural responses. Sites lacking ITD sensitivity (monaural) were associated with inputs primarily from cochlear nucleus and VNLL. Low-frequency, ITD-sensitive neurons were associated with dominant inputs from MSO and secondary inputs from ipsilateral LSO. Finally, neurons with BF above $3000 \mathrm{~Hz}$ and ITD sensitivity to complex signals like SAM tones, clicks, or noise, but not pure tones, were associated with inputs from both MSO and bilateral inputs from LSO. These results support the general notion that the ICC is organized into functional zones in which the responses of postsynaptic neurons in each zone are shaped by ascending inputs arising from a subset of subcollicular auditory nuclei.

\section{Implications for functional organization in ICC}

The present findings suggest at least three types of functional zones in the ICC of the cat. A monaural domain is dominated by inputs from the contralateral cochlear nucleus and ipsilateral VNLL. A low-frequency ITD domain is dominated by inputs from the ipsilateral MSO. Finally, a high-frequency ITD domain requires more complex stimuli and has more convergence from multiple superior olivary sources, including periolivary nuclei.

The "monaural domain" in the rostral and caudal ICCs contains neurons with unambiguous monaural responses and clearly differs from the two binaural domains on both physiological and anatomical grounds. The predominant input was from the $\mathrm{CN}$ and VNLL, regarded as predominantly monaural (Batra and Fitzpatrick, 1997; Shore et al., 2003; Sumner et al., 2005). This input pattern was not observed elsewhere in this dataset, suggesting that it is unique to monaural functional zones in the ICC. The monaural domain appears to be concentrated in the rostral and caudal ends of the ICC, and response properties are essentially shaped by the $\mathrm{CN}$ as suggested by Ramachandran et al. (Ramachandran et al., 1999; Ramachandran and May, 2002). VNLL may provide monaural, inhibitory modulation to this zone, because most VNLL neurons colocalize glycine and GABA (Riquelme et al., 1998). A monaural domain may be specialized for spectrotemporal processing (Escabí and Schreiner, 2002) or monaural spectral cues for location produced by directiondependent filtering by the cat's pinnae (Butler et al., 1990; Young et al., 1992; Delgutte et al., 1999; Imig et al., 2000; Poirier et al., 2003).

The "low-frequency $(<500 \mathrm{~Hz})$ ITD domain" in the dorsolateral ICC is marked by the prominent input from the ipsilateral MSO and the lateral, low-frequency limb of the iLSO. Because the iLSO is a likely source of glycinergic input (Saint Marie et al., 1989; Glendenning et al., 1992; Oliver et al., 1995), this projection may be the main inhibitory input to low-frequency ITD domain. Because the ITD in low-frequency signals is the most potent cue for the azimuthal position of low-frequency sounds (Wightman and Kistler, 1992), this functional zone may be designed for lowfrequency sound localization (Kuwada et al., 1979, 1987, 1997; Fitzpatrick et al., 1997; D’Angelo et al., 2003). The inhibitory LSO input could serve to sharpen the azimuthal receptive field (Kuwada et al., 1997). However, some projections from iLSO are glycinergic and may excite ICC neurons. The mixed peak and trough-type ITD responses in cases 24 and 176 of the present study support the notion of iLSO excitation.

The "high-frequency ITD domain" contained neurons with a variety of binaural responses. It is clearly the most heterogeneous of the three functional zones. Unlike the low-frequency ITD zone, this domain had a more varied and richer set of binaural inputs, including both MSO and bilateral LSO inputs. In addition, the DNLL was a frequent source of input and may be a primary source of inhibitory input for this domain (Shneiderman et al., 1988; Shneiderman and Oliver, 1989; Li and Kelly, 1992; Faingold et al., 1993), especially since at higher frequencies the iLSO glycinergic input is diminished (Saint Marie et al., 1989; Glendenning et al., 1992). The adequate stimuli were spectrally complex and included ITD sensitivity to envelopes in SAM tones and carriers in SAM tones and to click trains and noise. This is consistent with the notion that modulated stimuli are most important in this functional zone (Escabí et al., 2003, 2005; Zheng and Escabí, 2008) and most relevant to ITD coding at higher frequencies (Batra et al., 1989).

\section{The structure of functional zones in the ICC}

Our findings show reproducible anatomical patterns despite injections that are clearly larger than a single neuron. This is consistent with functional zones that are larger than a single neuron but smaller than the entire ICC. Because the center of our injection site is defined by the neural response at the tip of the electrode, there is an excellent correlation between the pattern of anatomical inputs and the response properties. However, the correlation may not be perfect, because the tracer may have diffused beyond the neuron at the center and may extend into an adjacent anatomical zone. This raises the question of how functional zones in the mammalian ICC are constructed. Clearly, it is different from the avian ICC, where different ascending inputs target different parts of ICC, each with its own tonotopic map (Takahashi and Konishi, 1988).

There are two different models for the structure of functional zones in the mammalian ICC. A functional zone in ICC may be related to the fibrodendritic lamina (Oliver et al., 1997; Malmierca et al., 2005). Alternatively, larger regions within the central nucleus may differ in their inputs. These may not be mutually exclusive organizations, because there is evidence for both types of organization. In both instances, when two different inputs project to different functional zones in IC, there should be at least one condition in which one input is labeled while the other is not.

The 150-200 $\mu \mathrm{m}$ wide fibrodendritic lamina contain closely spaced, disc-shaped neurons with parallel dendritic trees, and these neurons are likely to share the same inputs (Shneiderman and Henkel, 1987; Oliver et al., 1997; Loftus et al., 2004; Malmierca et al., 2005). Laminar axons in ICC tend to run long distances parallel to and within the dendritic laminae and give off only short branches or make en passant terminals, making it likely that single axons are confined to a single lamina (Oliver and Morest, 1984; Oliver et al., 1991). Anatomically, the fibrodendritic laminae may correspond to the narrowest dimension of the physiologically defined frequency band lamina and the discrete steps in the tonotopic organization seen in ICC (Schreiner and Langner, 1997; Malmierca et al., 2008). In most cases, our retrogradely 
labeled neurons in the lower auditory nuclei were confined to a single tonotopic position. Such a pattern is consistent with the BF of a small injection site confined to a single fibrodendritic lamina. However, previous experiments also showed tonotopically organized projections (Roth et al., 1978; Aitkin and Schuck, 1985; Maffi and Aitkin, 1987; Ross et al., 1988). But when only the tonotopic organization is considered, it may be unclear when an injection spreads to adjacent laminae. The adjacent laminae will code similar BFs, and after a larger injection a larger group of neurons will be labeled in adjacent positions in the lower auditory nuclei. This is problematic, because adjacent laminae may have different inputs.

Evidence that adjacent laminae in ICC may have different inputs comes primarily from the LSO of the cat (Shneiderman and Henkel, 1987; Saint Marie et al., 1989; Gabriele et al., 2007). Different neurons in LSO project to the ipsilateral and contralateral ICs, and their axons do not converge on the same target. Five animals in the present study (Table 1, cases 144, 151, 24, 176, 34) show labeling in only the ipsilateral LSO and MSO, with no labeling in the contralateral LSO. This is consistent with an injection confined to one lamina that receives inputs from the iLSO but not the cLSO. Only one experiment in each of three studies showed a similar result [case 76-4 in Roth et al. (1978), case 83-23 in Aitkin and Schuck (1985), and case 44 in Brunso-Bechtold et al. (1981)]. In contrast, injections that labeled both iLSO and cLSO are consistent with injections that label two adjacent laminae with different LSO inputs (Table 1). This was the common pattern in previous studies (Roth et al., 1978; Brunso-Bechtold et al., 1981; Maffi and Aitkin, 1987; Cant and Benson, 2006), but seen in only four of nine cases here with $\mathrm{BF}>1 \mathrm{kHz}$.

Larger regions of ICC also may have segregated inputs and serve as functional zones. In the present cases, injections that label only the cochlear nucleus and iVNLL were only in the extreme rostral and caudal ICs (Table 1). This is consistent with a physical separation of cochlear nucleus and superior olivary inputs also seen in the caudal IC of the gerbil (Cant and Benson, 2006).

The larger functional zones within ICC may be related to lowor high-frequency specialization. MSO is large in primates and other species capable of low-frequency ITD processing e.g. (Irving and Harrison, 1967; Masterton et al., 1975). Consequently, MSO neurons are the predominant group labeled after injections in the low-frequency IC (Roth et al., 1978; Aitkin and Schuck, 1985; Cant and Benson, 2006), a pattern supported in the present data (Table 1).

In contrast, the DCN is specialized for higher frequencies and lacks neurons with a BF below $1 \mathrm{kHz}$ in the cat (Spirou et al., 1993). This is consistent with our previous anterograde studies (Oliver, 1984) and a tendency to label DCN inputs when MSO and LSO labeling are absent or diminished (Maffi and Aitkin, 1987; Cant and Benson, 2006). A separation of DCN and superior olivary inputs is found in the present data despite undersampling of the DCN inputs.

The LSO projections may contribute to both laminar and larger organizations. Low BF LSO neurons tend to project to ipsilateral IC, and high-BF neurons project to contralateral IC (Glendenning and Masterton, 1983). These projections may terminate on more separated targets in low-frequency ICs, where iLSOs converge with MSO terminals (Loftus et al., 2004). In midfrequency IC, iLSO and cLSO laminae are side by side (Shneiderman and Henkel, 1987; Gabriele et al., 2007).

In summary, this study relates the function of ICC neurons with the anatomical circuitry that provides the synaptic inputs.
This methodological approach supports the synaptic domain hypothesis in that groups of adjacent ICC neurons appear to share the same pattern of inputs even in instances when only a single unit was isolated and studied. However, the higher frequency ITD-sensitive part of ICC is complex and is likely to contain multiple domains yet to be clarified. Here, there may be neurons in adjacent laminae with different patterns of inputs that produce different types of ITD sensitivity (Seshagiri and Delgutte, 2007). With our current methods, such neurons in adjacent laminae may be labeled with the same injection and, thus, blur a far more elegant synaptic domain organization than that seen here.

\section{References}

Aitkin L, Schuck D (1985) Low frequency neurons in the lateral central nucleus of the cat inferior colliculus receive their input predominantly from the medial superior olive. Hear Res 17:87-93.

Batra R, Fitzpatrick DC (1997) Neurons sensitive to interaural temporal disparities in the medial part of the ventral nucleus of the lateral lemniscus. J Neurophysiol 78:511-515.

Batra R, Fitzpatrick DC (2002) Processing of interaural temporal disparities in the medial division of the ventral nucleus of the lateral lemniscus. J Neurophysiol 88:666-675.

Batra R, Kuwada S, Stanford TR (1989) Temporal coding of envelopes and their interaural delays in the inferior colliculus of the unanesthetized rabbit. J Neurophysiol 61:257-268.

Batra R, Kuwada S, Stanford TR (1993) High-frequency neurons in the inferior colliculus that are sensitive to interaural delays of amplitudemodulated tones: evidence for dual binaural influences. J Neurophysiol 70:64-80.

Batra R, Kuwada S, Fitzpatrick DC (1997) Sensitivity to interaural temporal disparitiees of low- and high-frequency neurons in the superior olivary complex: I. Heterogeneity of responses. J Neurophysiol 78:1222-1236.

Brunso-Bechtold JK, Thompson GC, Masterton RB (1981) HRP study of the organization of auditory afferents ascending to central nucleus of inferior colliculus in cat. J Comp Neurol 197:705-722.

Butler RA, Humanski RA, Musicant AD (1990) Binaural and monaural localization of sound in two-dimensional space. Perception 19:241-256.

Cant NB, Benson CG (2006) Organization of the inferior colliculus of the gerbil (Meriones unguiculatus): differences in distribution of projections from the cochlear nuclei and the superior olivary complex. J Comp Neurol 495:511-528.

D’Angelo WR, Sterbing SJ, Ostapoff EM, Kuwada S (2003) Effects of amplitude modulation on the coding of interaural time differences of lowfrequency sounds in the inferior colliculus. II. Neural mechanisms. J Neurophysiol 90:2827-2836.

D’Angelo WR, Sterbing SJ, Ostapoff EM, Kuwada S (2005) Role of GABAergic inhibition in the coding of interaural time differences of low-frequency sounds in the inferior colliculus. J Neurophysiol 93:3390-3400.

Davis KA, Ramachandran R, May BJ (1999) Single-unit responses in the inferior colliculus of decerebrate cats II. Sensitivity to interaural level differences. J Neurophysiol 82:164-175.

Delgutte B, Joris PX, Litovsky RY, Yin TC (1999) Receptive fields and binaural interactions for virtual-space stimuli in the cat inferior colliculus. J Neurophysiol 81:2833-2851.

Escabí MA, Schreiner CE (2002) Nonlinear spectrotemporal sound analysis by neurons in the auditory midbrain. J Neurosci 22:4114-4131.

Escabí MA, Miller LM, Read HL, Schreiner CE (2003) Naturalistic auditory contrast improves spectrotemporal coding in the cat inferior colliculus. J Neurosci 23:11489-11504.

Escabí MA, Nassiri R, Miller LM, Schreiner CE, Read HL (2005) The contribution of spike threshold to acoustic feature selectivity, spike information content, and information throughput. J Neurosci 25:9524-9534.

Faingold CL, Anderson CA, Randall ME (1993) Stimulation or blockade of the dorsal nucleus of the lateral lemniscus alters binaural and tonic inhibition in contralateral inferior colliculus neurons. Hear Res 69:98-106.

Fitzpatrick DC, Batra R, Stanford TR, Kuwada S (1997) A neuronal population code for sound localization. Nature 388:871-874

Fitzpatrick DC, Kuwada S, Batra R (2000) Neural sensitivity to interaural time differences: beyond the Jeffress model. J Neurosci 20:1605-1615.

Fitzpatrick DC, Roberts JM, Kuwada S, Kim DO, Filipovic B (2009) Processing temporal modulations in binaural and monaural auditory stimuli 
by neurons in the inferior colliculus and auditory cortex. J Assoc Res Otolaryngol 10:579-593.

Gabriele ML, Shahmoradian SH, French CC, Henkel CK, McHaffie JG (2007) Early segregation of layered projections from the lateral superior olivary nucleus to the central nucleus of the inferior colliculus in the neonatal cat. Brain Res 1173:66-77.

Glendenning KK, Masterton RB (1983) Acoustic chiasm: efferent projections of the lateral superior olive. J Neurosci 3:1521-1537.

Glendenning KK, Baker BN, Hutson KA, Masterton RB (1992) Acoustic chiasm V: inhibition and excitation in the ipsilateral and contralateral projections of LSO. J Comp Neurol 319:100-122.

Imig TJ, Bibikov NG, Poirier P, Samson FK (2000) Directionality derived from pinna-cue spectral notches in cat dorsal cochlear nucleus. J Neurophysiol 83:907-925.

Irving R, Harrison JM (1967) The superior olivary complex and audition: a comparative study. J Comp Neurol 130:77-86.

Joris PX, Yin TC (1995) Envelope coding in the lateral superior olive. I. Sensitivity to interaural time differences. J Neurophysiol 73:1043-1062.

Kuwada S, Yin TC, Wickesberg RE (1979) Response of cat inferior colliculus neurons to binaural beat stimuli: possible mechanisms for sound localization. Science 206:586-588.

Kuwada S, Stanford TR, Batra R (1987) Interaural phase-sensitive units in the inferior colliculus of the unanesthetized rabbit: effects of changing frequency. J Neurophysiol 57:1338-1360.

Kuwada S, Batra R, Stanford TR (1989) Monaural and binaural response properties of neurons in the inferior colliculus of the rabbit: effects of sodium pentobarbital. J Neurophysiol 61:269-282.

Kuwada S, Batra R, Fitzpatrick DC (1997) Neural processing of binaural temporal cues. In: Binaural and spatial hearing in real and virtual environments (Gilkey RH, Anderson TR, eds), pp 399-425. Mahwah, NJ: Erlbaum.

Kuwada S, Fitzpatrick DC, Batra R, Ostapoff EM (2006) Sensitivity to interaural time differences in the dorsal nucleus of the lateral lemniscus of the unanesthetized rabbit: comparison with other structures. J Neurophysiol 95:1309-1322.

Li L, Kelly JB (1992) Inhibitory influence of the dorsal nucleus of the lateral lemniscus on binaural responses in the rat's inferior colliculus. J Neurosci $12: 4530-4539$.

Loftus WC, Bishop DC, Saint Marie RL, Oliver DL (2004) Organization of binaural excitatory and inhibitory inputs to the inferior colliculus from the superior olive. J Comp Neurol 472:330-344.

Loftus WC, Bishop DC, Oliver DL (2006) Spatially divergent patterns of input from lower auditory nuclei contribute to functional specificity in the inferior colliculus (IC). Soc Neurosci Abstr 32:544.7.

Loftus WC, Malmierca MS, Bishop DC, Oliver DL (2008) The cytoarchitecture of the inferior colliculus revisited: a common organization of the lateral cortex in rat and cat. Neuroscience 154:196-205.

Maffi CL, Aitkin LM (1987) Differential neural projections to regions of the inferior colliculus of the cat responsive to high frequency sounds. Hear Res 26:211-219.

Malmierca MS, Hackett TA (2010) Structural organization of the ascending auditory pathway. In: The Oxford handbook of auditory science: the auditory brain (Rees A, Palmer AR, eds), pp 9-42. Oxford: Oxford UP.

Malmierca MS, Saint Marie RL, Merchan MA, Oliver DL (2005) Laminar inputs from dorsal cochlear nucleus and ventral cochlear nucleus to the central nucleus of the inferior colliculus: two patterns of convergence. Neuroscience 136:883-894.

Malmierca MS, Izquierdo MA, Cristaudo S, Hernández O, Pérez-González D, Covey E, Oliver DL (2008) A discontinuous tonotopic organization in the inferior colliculus of the rat. J Neurosci 28:4767-4776.

Malone BJ, Schreiner CE (2010) Time-varying sounds: amplitude envelope modulations. In: The Oxford handbook of auditory science: the auditory brain (Rees A, Palmer AR, eds), pp 125-148. Oxford: Oxford UP.

Masterton B, Thompson GC, Bechtold JK, RoBards MJ (1975) Neuroanatomical basis of binaural phase-difference analysis for sound localization: a comparative study. J Comp Physiol Psychol 89:379-386.

Merchán MA, Berbel P (1996) Anatomy of the ventral nucleus of the lateral lemniscus in rats: a nucleus with a concentric laminar organization. J Comp Neurol 372:245-263.

Morest DK, Oliver DL (1984) The neuronal architecture of the inferior colliculus in the cat: defining the functional anatomy of the auditory midbrain. J Comp Neurol 222:209-236.
Oliver DL (1984) Dorsal cochlear nucleus projections to the inferior colliculus in the cat: a light and electron microscopic study. J Comp Neurol 224:155-172.

Oliver DL (2000) Ascending efferent projections of the superior olivary complex. Microsc Res Tech 51:355-363.

Oliver DL (2005) Neuronal organization in the inferior colliculus. In: The inferior colliculus. New York: Springer.

Oliver DL, Huerta MF (1992) Inferior and superior colliculi. In: The mammalian auditory pathway: neuroanatomy, 1st Ed (Webster DB, Popper AN, Fay RR, eds), pp 168-221. New York: Springer.

Oliver DL, Morest DK (1984) The central nucleus of the inferior colliculus in the cat. J Comp Neurol 222:237-264.

Oliver DL, Kuwada S, Yin TC, Haberly LB, Henkel CK (1991) Dendritic and axonal morphology of HRP-injected neurons in the inferior colliculus of the cat. J Comp Neurol 303:75-100.

Oliver DL, Beckius GE, Shneiderman A (1995) Axonal projections from the lateral and medial superior olive to the inferior colliculus of the cat: a study using electron microscopic autoradiography. J Comp Neurol 360:17-32.

Oliver DL, Beckius GE, Bishop DC, Kuwada S (1997) Simultaneous anterograde labeling of axonal layers from lateral superior olive and dorsal cochlear nucleus in the inferior colliculus of cat. J Comp Neurol 382:215-229.

Oliver DL, Beckius GE, Bishop DC, Loftus WC, Batra R (2003) Topography of interaural temporal disparity coding in projections of medial superior olive to inferior colliculus. J Neurosci 23:7438-7449.

Pieribone VA, Aston-Jones G (1988) The iontophoretic application of Fluoro-Gold for the study of afferents to deep brain nuclei. Brain Res 475:259-271.

Poirier P, Samson FK, Imig TJ (2003) Spectral shape sensitivity contributes to the azimuth tuning of neurons in the cat's inferior colliculus. J Neurophysiol 89:2760-2777.

Ramachandran R, May BJ (2002) Functional segregation of ITD sensitivity in the inferior colliculus of decerebrate cats. J Neurophysiol 88:2251-2261.

Ramachandran R, Davis KA, May BJ (1999) Single-unit responses in the inferior colliculus of decerebrate cats I. Classification based on frequency response maps. J Neurophysiol 82:152-163.

Riquelme R, Merchan MA, Ottersen OP (1998) GABA and glycine in the ventral nucleus of the lateral lemniscus: an immunocytochemical and in situ hybridization study in rat. Assoc Res Otolaryngol Abstr 21:93.

Rose JE, Gross NB, Geisler CD, Hind JE (1966) Some neural mechanisms in the inferior colliculus of the cat which may be relevant to localization of a sound source. J Neurophysiol 29:288-314.

Ross LS, Pollak GD, Zook JM (1988) Origin of ascending projections to an isofrequency region of the mustache bat's inferior colliculus. J Comp Neurol 270:488-505.

Roth GL, Aitkin LM, Andersen RA, Merzenich MM (1978) Some features of the spatial organization of the central nucleus of the inferior colliculus of the cat. J Comp Neurol 182:661-680.

Saint Marie RL, Ostapoff EM, Morest DK, Wenthold RJ (1989) Glycineimmunoreactive projection of the cat lateral superior olive: possible role in midbrain ear dominance. J Comp Neurol 279:382-396.

Schmued LC, Fallon JH (1986) Fluoro-Gold: a new fluorescent retrograde axonal tracer with numerous unique properties. Brain Res 377:147-154.

Schreiner CE, Langner G (1997) Laminar fine structure of frequency organization in auditory midbrain. Nature 388:383-386.

Semple MN, Aitkin LM (1979) Representation of sound frequency and laterality by units in central nucleus of cat inferior colliculus. J Neurophysiol 42:1626-1639.

Seshagiri CV, Delgutte B (2007) Response properties of neighboring neurons in the auditory midbrain for pure-tone stimulation: a tetrode study. J Neurophysiol 98:2058-2073.

Shneiderman A, Henkel CK (1987) Banding of lateral superior olivary nucleus afferents in the inferior colliculus: A possible substrate for sensory integration. J Comp Neurol 266:519-534.

Shneiderman A, Oliver DL (1989) EM autoradiographic study of the projections from the dorsal nucleus of the lateral lemniscus: a possible source of inhibitory inputs to the inferior colliculus. J Comp Neurol 286:28 -47.

Shneiderman A, Oliver DL, Henkel CK (1988) Connections of the dorsal nucleus of the lateral lemniscus: an inhibitory parallel pathway in the ascending auditory system? J Comp Neurol 276:188-208.

Shore SE, Sumner CJ, Bledsoe SC, Lu J (2003) Effects of contralateral sound 
stimulation on unit activity of ventral cochlear nucleus neurons. Exp Brain Res 153:427-435.

Spirou GA, May BJ, Wright DD, Ryugo DK (1993) Frequency organization of the dorsal cochlear nucleus in cats. J Comp Neurol 329:36-52.

Sterbing SJ, D'Angelo WR, Ostapoff EM, Kuwada S (2003) Effects of amplitude modulation on the coding of interaural time differences of lowfrequency sounds in the inferior colliculus. I. Response properties. J Neurophysiol 90:2818-2826.

Sumner CJ, Tucci DL, Shore SE (2005) Responses of ventral cochlear nucleus neurons to contralateral sound after conductive hearing loss. J Neurophysiol 94:4234-4243.

Takahashi TT, Konishi M (1988) Projections of the cochlear nuclei and nucleus laminaris to the inferior colliculus of the barn owl. J Comp Neurol 274:190-211.

Wightman FL, Kistler DJ (1992) The dominant role of low-frequency interaural time differences in sound localization. J Acoust Soc Am 91:1648-1661.
Yin TC, Kuwada S (1983) Binaural interaction in low-frequency neurons in inferior colliculus of the cat. II. Effects of changing rate and direction of interaural phase. J Neurophysiol 50:1000-1019.

Yin TC, Kuwada S (2010) Binaural localization cues. In: The Oxford handbook of Auditory science: the auditory brain (Rees A, Palmer AR, eds), pp 271-302. Oxford: Oxford UP.

Young ED (2010) Level and spectrum. In: The Oxford handbook of auditory science: the auditory brain (Rees A, Palmer AR, eds), pp 93-124. Oxford: Oxford UP.

Young ED, Spirou GA, Rice JJ, Voigt HF (1992) Neural organization and responses to complex stimuli in the dorsal cochlear nucleus. Philos Trans R Soc Lond B Biol Sci 336:407-413.

Zheng Y, Escabí MA (2008) Distinct roles for onset and sustained activity in the neuronal code for temporal periodicity and acoustic envelope shape. J Neurosci 28:14230-14244. 